\title{
Isoperimetric inequalities for eigenvalues of the Laplacian and the Schrödinger operator
}

\author{
Rafael D. Benguria • Helmut Linde • \\ Benjamín Loewe
}

Received: 18 April 2011 / Revised: 18 October 2011 / Accepted: 20 November 2011 /

Published online: 10 January 2012

(C) The Author(s) 2012. This article is published with open access at SpringerLink.com

\begin{abstract}
The purpose of this manuscript is to present a series of lecture notes on isoperimetric inequalities for the Laplacian, for the Schrödinger operator, and related problems.
\end{abstract}

Keywords Eigenvalues of the Laplacian · Isoperimetric inequalities

Mathematics Subject Classification (1991) Primary 35P15; Secondary 35J05 . 49R50

\section{Introduction}

They came to this place, and bought land, where you now see the vast walls, and resurgent stronghold, of new Carthage, as much as they could enclose with the strips of hide from a single bull, and from that they called it Byrsa. Book I: "The Trojans reach Carthage", in The Aeneid.

Communicated by A. Laptev.

R. D. Benguria $(\varangle) \cdot$ B. Loewe

Departamento de Física, P. Universidad Católica de Chile,

Casilla 306, Santiago 22, Chile

e-mail: rbenguri@fis.puc.cl

B. Loewe

e-mail: baloewe@puc.cl

H. Linde

Global Management Office, Field Services and Support,

SAP Deutschland AG. \& Co. KG, Walldorf, Germany

e-mail: helmut.linde@sap.com 
Isoperimetric inequalities have played an important role in mathematics since the times of ancient Greece. The first and best known isoperimetric inequality is the classical isoperimetric inequality

$$
A \leq \frac{L^{2}}{4 \pi},
$$

relating the area $A$ enclosed by a planar closed curve of perimeter $L$. In mathematics, the oldest existing recorded appearance of this problem is in Book V, The Sagacity of Bees, in the "Collection" of Pappus of Alexandria (IV century AD). The whole of Book V in Pappus Collection is devoted to isoperimetry. The first section follows closely the previous exposition of Zenodorus (now lost) as recounted by Theon (see [101]), where Pappus proposes the two problems: i) Which plane figure of a given perimeter encloses the largest area? and also ii) Which solid figure with given surface area encloses the largest volume? [59]. The first problem proposed by Pappus is widely known as Queen Dido's problem in connection to the foundation of Carthage, described in Virgilio's epic poem "The Aeneid" [102]). Previously, Euclid in his Elements (Book VI) had solved the related simpler problem, of all rectangles of a given perimeter, the one that encloses the largest area is the square. This problem, solved geometrically by Euclid, is equivalent to the well known inequality between the geometric and the arithmetic mean. After the introduction of Calculus in the XVII century, many new isoperimetric inequalities have been discovered in mathematics and physics (see, e.g., the review articles [20,80,82,87]; see also the essay [16] for a panorama on the subject of Isoperimetry). The eigenvalues of the Laplacian are "geometric objects" in the sense they do depend on the geometry of the underlying domain, and to some extent (see Section 2) the knowledge of the domain characterizes the geometry of the domain. Therefore it is natural to pose the problem of finding isoperimetric inequalities for the eigenvalues of the Laplacian. The first one to consider this possibility was Lord Rayleigh in his monograph The Theory of Sound [88]. In these lectures we will present some of the problems arising in the study of isoperimetric inequalities for the Laplacian, some of the tools needed in their proof and many bibliographic discussions about the subject. We start our review with the classical problem of Mark Kac, Can one hear the shape of a drum. In Section 3 we review the definitions and basic facts about rearrangements of functions. Section 4 is devoted to the Rayleigh-Faber-Krahn inequality. In Section 5 we review the Szegö-Weinberger inequality, which is an isoperimetric inequality for the first nontrivial Neumann eigenvalue of the Laplacian. In Section 6 we review the Payne-Pólya-Weinberger isoperimetric inequality for the quotient of the first two Dirichlet eigenvalues of the Laplacian, as well as several recent extensions. In Section 7, we review the "Fundamental Gap Formula" between the lowest two Dirichlet eigenvalues for a bounded, convex domain in $\mathbb{R}^{N}$, which was recently proved by Andrews and Clutterbuck [1]. In Section 8, we review an interesting conjectured isoperimetric property for ovals in the plane, which arises in connection with the Lieb-Thirring inequalities. Finally, in Section 9 we review three different isoperimetric problems for fourth order operators. There are many interesting results that we have left out of this review. For different perspectives, selections and emphasis, please refer for example to the reviews $[3-5,15,60]$, among many others. 
The contents of this manuscript are an extended version of the lectures that one of us (RB) gave as an intensive course for graduate students in the Tunis Science City, Tunisia (May 21-22, 2010), in connection with the International Conference on the isoperimetric problem of Queen Dido and its mathematical ramifications, that was held in Carthage, Tunisia, May 24-29, 2010. We would like to thank all the local organizers of the conference, especially Najoua Gamara and Lotfi Hermi, for their great hospitality. Previous versions of this intensive course have been given by RB in the Arizona School of Analysis with Applications, University of Arizona, Tucson, AZ, March 15-19, 2010, and previously in the IV Escuela de Verano en Análisis y Física Matemática at the Unidad Cuernavaca del Instituto de Matemáticas de la Universidad Nacional Autónoma de México, in the summer of 2005 [25]. Preliminary versions of these lectures were also given in the Short Course in Isoperimetric Inequalities for Eigenvalues of the Laplacian, given by one of us (RB) in February of 2004, as part of the Thematic Program on Partial Differential Equations held at the Fields Institute, in Toronto, and also as part of the course Autovalores del Laplaciano y Geometría given at the Department of Mathematics of the Universidad de Pernambuco, in Recife, Brazil, in August 2003.

\title{
2 Can one hear the shape of a drum?
}

\author{
...but it would baffle the most skillful \\ mathematician to solve the Inverse Problem, \\ and to find out the shape of a bell by means \\ of the sounds which is capable of sending out \\ Sir Arthur Schuster (1882).
}

In 1965, the Committee on Educational Media of the Mathematical Association of America produced a film on a mathematical lecture by Mark Kac (1914-1984) with the title: Can one hear the shape of a drum? One of the purposes of the film was to inspire undergraduates to follow a career in mathematics. An expanded version of that lecture was later published [62]. Consider two different smooth, bounded domains, say $\Omega_{1}$ and $\Omega_{2}$ in the plane. Let $0<\lambda_{1}<\lambda_{2} \leq \lambda_{3} \leq \ldots$ be the sequence of eigenvalues of the Laplacian on $\Omega_{1}$, with Dirichlet boundary conditions and, correspondingly, $0<\lambda_{1}^{\prime}<\lambda_{2}^{\prime} \leq \lambda_{3}^{\prime} \leq \ldots$ be the sequence of Dirichlet eigenvalues for $\Omega_{2}$. Assume that for each $n, \lambda_{n}=\lambda_{n}^{\prime}$ (i.e., both domains are isospectral). Then, Mark Kac posed the following question: Are the domains $\Omega_{1}$ and $\Omega_{2}$ congruent in the sense of Euclidean geometry?. A friend of Mark Kac, the mathematician Lipman Bers (1914-1993), paraphrased this question in the famous sentence: Can one hear the shape of a drum?

In 1910, H. A. Lorentz, at the Wolfskehl lecture at the University of Göttingen, reported on his work with Jeans on the characteristic frequencies of the electromagnetic field inside a resonant cavity of volume $\Omega$ in three dimensions. According to the work of Jeans and Lorentz, the number of eigenvalues of the electromagnetic cavity whose numerical values is below $\lambda$ (this is a function usually denoted by $N(\lambda)$ ) is given asymptotically by

$$
N(\lambda) \approx \frac{|\Omega|}{6 \pi^{2}} \lambda^{3 / 2}
$$


for large values of $\lambda$, for many different cavities with simple geometry (e.g., cubes, spheres, cylinders, etc.) Thus, according to the calculations of Jeans and Lorentz, to leading order in $\lambda$, the counting function $N(\lambda)$ seemed to depend only on the volume of the electromagnetic cavity $|\Omega|$. Apparently David Hilbert (1862-1943), who was attending the lecture, predicted that this conjecture of Lorentz would not be proved during his lifetime. This time, Hilbert was wrong, since his own student, Hermann Weyl (1885-1955) proved the conjecture less than two years after the Lorentz' lecture.

Remark There is a nice account of the work of Hermann Weyl on the eigenvalues of a membrane in his 1948 J. W. Gibbs Lecture to the American Mathematical Society [105].

In $N$ dimensions, (2.1) reads,

$$
N(\lambda) \approx \frac{|\Omega|}{(2 \pi)^{N}} C_{N} \lambda^{N / 2},
$$

where $\left.C_{N}=\pi^{(N / 2)} / \Gamma((N / 2)+1)\right)$ denotes the volume of the unit ball in $N$ dimensions.

Using Tauberian theorems, one can relate the behavior of the counting function $N(\lambda)$ for large values of $\lambda$ with the behavior of the function

$$
Z_{\Omega}(t) \equiv \sum_{n=1}^{\infty} \exp \left\{-\lambda_{n} t\right\}
$$

for small values of $t$. The function $Z_{\Omega}(t)$ is the trace of the heat kernel for the domain $\Omega$, i.e., $Z_{\Omega(t)}=\operatorname{tr} \exp (\Delta t)$. As we mention above, $\lambda_{n}(\Omega)$ denotes the $n$ Dirichlet eigenvalue of the domain $\Omega$.

\section{An example: the behavior of $Z_{\Omega}(t)$ for rectangles.}

With the help of the Riemann Theta function $\Theta(x)$, it is simple to compute the trace of the heat kernel when the domain is a rectangle of sides $a$ and $b$, and therefore to obtain the leading asymptotic behavior for small values of $t$. The Riemann Theta function is defined by

$$
\Theta(x)=\sum_{n=-\infty}^{\infty} e^{-\pi n^{2} x},
$$

for $x>0$. The function $\Theta(x)$ satisfies the following modular relation,

$$
\Theta(x)=\frac{1}{\sqrt{x}} \Theta\left(\frac{1}{x}\right) .
$$

Remark This modular form for the Theta Function already appears in the classical paper of Riemann [91] (manuscript where Riemann puts forward his famous Riemann Hypothesis). In that manuscript, the modular form (2.5) is attributed to Jacobi. 
The modular form (2.5) may be obtained from a very elegant application of Fourier Analysis (see, e.g., [43], pp. 75-76) which we reproduce here for completeness. Define

$$
\varphi_{x}(y)=\sum_{n=-\infty}^{\infty} e^{-\pi(n+y)^{2} x} .
$$

Clearly, $\Theta(x)=\varphi_{x}(0)$. Moreover, the function $\varphi_{x}(y)$ is periodic in $y$ of period 1 . Thus, we can express it as follows,

$$
\varphi_{x}(y)=\sum_{k=-\infty}^{\infty} a_{k} e^{2 \pi k i y}
$$

where, the Fourier coefficients are

$$
a_{k}=\int_{0}^{1} \varphi_{k}(y) e^{-2 \pi k i y} d y .
$$

Replacing the expression (2.6) for $\varphi_{x}(y)$ in (2.9), using the fact that $e^{2 \pi k i n}=1$, we can write,

$$
a_{k}=\int_{0}^{1} \sum_{n=-\infty}^{\infty} e^{-\pi(n+y)^{2} x} e^{-2 \pi i k(y+n)} d y .
$$

Interchanging the order between the integral and the sum, we get,

$$
a_{k}=\sum_{n=-\infty}^{\infty} \int_{0}^{1}\left(e^{-\pi(n+y)^{2} x} e^{-2 \pi i k(y+n)}\right) d y \text {. }
$$

In the $n$th summand we make the change of variables $y \rightarrow u=n+y$. Clearly, $u$ runs from $n$ to $n+1$, in the $n$th summand. Thus, we get,

$$
a_{k}=\int_{-\infty}^{\infty} e^{-\pi u^{2} x} e^{-2 \pi i k u} d u
$$

i.e., $a_{k}$ is the Fourier transform of a Gaussian. Thus, we finally obtain,

$$
a_{k}=\frac{1}{\sqrt{x}} e^{-\pi k^{2} / x}
$$

Since, $\Theta(x)=\varphi_{x}(0)$, from (2.7) and (2.12) we finally get,

$$
\Theta(x)=\sum_{k=-\infty}^{\infty} a_{k}=\frac{1}{\sqrt{x}} \sum_{k=-\infty}^{\infty} e^{-\pi k^{2} / x}=\frac{1}{\sqrt{x}} \Theta\left(\frac{1}{x}\right) .
$$


Remarks i) The method exhibited above is a particular case of the Poisson Summation Formula (see, e.g., [43], pp. 76-77); ii) It should be clear from (2.4) that $\lim _{x \rightarrow \infty} \Theta(x)=1$. Hence, from the modular form for $\Theta(x)$ we immediately see that

$$
\lim _{x \rightarrow 0} \sqrt{x} \Theta(x)=1
$$

Once we have the modular form for the Riemann Theta function, it is simple to get the leading asymptotic behavior of the trace of the heat kernel $Z_{\Omega}(t)$, for small values of $t$, when the domain $\Omega$ is a rectangle. Take $\Omega$ to be the rectangle of sides $a$ and $b$. Its Dirichlet eigenvalues are given by

$$
\lambda_{n, m}=\pi^{2}\left[\frac{n^{2}}{a^{2}}+\frac{m^{2}}{b^{2}}\right],
$$

with $n, m=1,2, \ldots$ In terms of the Dirichlet eigenvalues, the trace of the heat kernel, $Z_{\Omega}(t)$ is given by

$$
Z_{\Omega}(t)=\sum_{n, m=1}^{\infty} e^{-\lambda_{n, m} t}
$$

and using (2.15), and the definition of $\Theta(x)$, we get,

$$
Z_{\Omega}(t)=\frac{1}{4}\left[\theta\left(\frac{\pi t}{a^{2}}\right)-1\right]\left[\theta\left(\frac{\pi t}{b^{2}}\right)-1\right] .
$$

Using the asymptotic behavior of the Theta function for small arguments, i.e., (2.14) above, we have

$$
Z_{\Omega}(t) \approx \frac{1}{4}\left(\frac{a}{\sqrt{\pi t}}-1\right)\left(\frac{b}{\sqrt{\pi t}}-1\right) \approx \frac{1}{4 \pi t} a b-\frac{1}{4 \sqrt{\pi t}}(a+b)+\frac{1}{4}+O(t) .
$$

In terms of the area of the rectangle $A=a b$ and its perimeter $L=2(a+b)$, the expression $Z_{\Omega}(t)$ for the rectangle may be written simply as,

$$
Z_{\Omega}(t) \approx \frac{1}{4 \pi t} A-\frac{1}{8 \sqrt{\pi t}} L+\frac{1}{4}+O(t)
$$

Remark Using similar techniques, one can compute the small $t$ behavior of $Z_{\Omega}(t)$ for various simple regions of the plane (see, e.g., [73]).

The fact that the leading behavior of $Z_{\Omega}(t)$ for $t$ small, for any bounded, smooth domain $\Omega$ in the plane is given by

$$
Z_{\Omega}(t) \approx \frac{1}{4 \pi t} A
$$


was proven by Hermann Weyl [104]. Here, $A=|\Omega|$ denotes the area of $\Omega$. In fact, what Weyl proved in [104] is the Weyl Asymptotics of the Dirichlet eigenvalues, i.e., for large $n, \lambda_{n} \approx(4 \pi n) / A$. Weyl's result (2.20) implies that one can hear the area of the drum.

In 1954, the Swedish mathematician Åke Pleijel [86] obtained the improved asymptotic formula,

$$
Z(t) \approx \frac{A}{4 \pi t}-\frac{L}{8 \sqrt{\pi} t},
$$

where $L$ is the perimeter of $\Omega$. In other words, one can hear the area and the perimeter of $\Omega$. It follows from Pleijel's asymptotic result that if all the frequencies of a drum are equal to those of a circular drum then the drum must itself be circular. This follows from the classical isoperimetric inequality (i.e., $L^{2} \geq 4 \pi A$, with equality if and only if $\Omega$ is a circle). In other words, one can hear whether a drum is circular. It turns out that it is enough to hear the first two eigenfrequencies to determine whether the drum has the circular shape [9].

In 1966, Mark Kac obtained the next term in the asymptotic behavior of $Z(t)$ [62]. For a smooth, bounded, multiply connected domain on the plane (with $r$ holes)

$$
Z(t) \approx \frac{A}{4 \pi t}-\frac{L}{8 \sqrt{\pi} t}+\frac{1}{6}(1-r) .
$$

Thus, one can hear the connectivity of a drum. The last term in the above asymptotic expansion changes for domains with corners (e.g., for a rectangular membrane, using the modular formula for the Theta Function, we obtained 1/4 instead of 1/6). Kac's formula (2.21) was rigorously justified by McKean and Singer [74]. Moreover, for domains having corners they showed that each corner with interior angle $\gamma$ makes an additional contribution to the constant term in $(2.21)$ of $\left(\pi^{2}-\gamma^{2}\right) /(24 \pi \gamma)$. (Notice that for a rectangle, i.e., when all the corners have $\gamma=\pi / 2$, this factor is $1 / 16$, which multiplied by 4 , i.e., by the number of corners, yields the third term in (2.19)).

A sketch of Kac's analysis for the first term of the asymptotic expansion is as follows (here we follow [62,73]). If we imagine some substance concentrated at $\vec{\rho}=\left(x_{0}, y_{0}\right)$ diffusing through the domain $\Omega$ bounded by $\partial \Omega$, where the substance is absorbed at the boundary, then the concentration $P_{\Omega}(\vec{p} \mid \vec{r} ; t)$ of matter at $\vec{r}=(x, y)$ at time $t$ obeys the diffusion equation

$$
\frac{\partial P_{\Omega}}{\partial t}=\Delta P_{\Omega}
$$

with boundary condition $P_{\Omega}(\vec{p} \mid \vec{r} ; t) \rightarrow 0$ as $\vec{r} \rightarrow \vec{a}, \vec{a} \in \partial \Omega$, and initial condition $P_{\Omega}(\vec{p} \mid \vec{r} ; t) \rightarrow \delta(\vec{r}-\vec{p})$ as $t \rightarrow 0$, where $\delta(\vec{r}-\vec{p})$ is the Dirac delta function. The concentration $P_{\Omega}(\vec{p} \mid \vec{r} ; t)$ may be expressed in terms of the Dirichlet eigenvalues of $\Omega, \lambda_{n}$ and the corresponding (normalized) eigenfunctions $\phi_{n}$ as follows,

$$
P_{\Omega}(\vec{p} \mid \vec{r} ; t)=\sum_{n=1}^{\infty} e^{-\lambda_{n} t} \phi_{n}(\vec{p}) \phi_{n}(\vec{r}) .
$$


For small $t$, the diffusion is slow, that is, it will not feel the influence of the boundary in such a short time. We may expect that

$$
P_{\Omega}(\vec{p} \mid \vec{r} ; t) \approx P_{0}(\vec{p} \mid \vec{r} ; t),
$$

ar $t \rightarrow 0$, where $\partial P_{0} / \partial t=\Delta P_{0}$, and $P_{0}(\vec{p} \mid \vec{r} ; t) \rightarrow \delta(\vec{r}-\vec{p})$ as $t \rightarrow 0 . P_{0}$ in fact represents the heat kernel for the whole $\mathbb{R}^{2}$, i.e., no boundaries present. This kernel is explicitly known. In fact,

$$
P_{0}(\vec{p} \mid \vec{r} ; t)=\frac{1}{4 \pi t} \exp \left(-|\vec{r}-\vec{p}|^{2} / 4 t\right)
$$

where $|\vec{r}-\vec{p}|^{2}$ is just the Euclidean distance between $\vec{p}$ and $\vec{r}$. Then, as $t \rightarrow 0^{+}$,

$$
P_{\Omega}(\vec{p} \mid \vec{r} ; t)=\sum_{n=1}^{\infty} e^{-\lambda_{n} t} \phi_{n}(\vec{p}) \phi_{n}(\vec{r}) \approx \frac{1}{4 \pi t} \exp \left(-|\vec{r}-\vec{p}|^{2} / 4 t\right)
$$

Thus, when set $\vec{p}=\vec{r}$ we get

$$
\sum_{n=1}^{\infty} e^{-\lambda_{n} t} \phi_{n}^{2}(\vec{r}) \approx \frac{1}{4 \pi t}
$$

Integrating both sides with respect to $\vec{r}$, using the fact that $\phi_{n}$ is normalized, we finally get,

$$
\sum_{n=1}^{\infty} e^{-\lambda_{n} t} \approx \frac{|\Omega|}{4 \pi t}
$$

which is the first term in the expansion (2.21). Further analysis gives the remaining terms (see [62]).

Remark In 1951, Mark Kac proved [61] the following universal bound on $Z(t)$ in dimension $d$ :

$$
Z(t) \leq \frac{|\Omega|}{(4 \pi t)^{d / 2}}
$$

This bound is sharp, in the sense that as $t \rightarrow 0$,

$$
Z(t) \approx \frac{|\Omega|}{(4 \pi t)^{d / 2}}
$$

Recently, Harrell and Hermi [58] proved the following improvement on (2.24),

$$
Z(t) \approx \frac{|\Omega|}{(4 \pi t)^{d / 2}} e^{-M_{d}|\Omega| t / I(\Omega)} .
$$


where $I_{\Omega}=\min _{a \in \mathbb{R}^{d}} \int_{\Omega}|x-a|^{2} d x$ and $M_{d}$ is a constant depending on dimension. Moreover, they conjectured the following bound on $Z(t)$, namely,

$$
Z(t) \approx \frac{|\Omega|}{(4 \pi t)^{d / 2}} e^{-t /|\Omega|^{2 / d}}
$$

Recently, Geisinger and Weidl [52] proved the best bound up to date in this direction,

$$
Z(t) \approx \frac{|\Omega|}{(4 \pi t)^{d / 2}} e^{-\bar{M}_{d} t /|\Omega|^{2 / d}},
$$

where $\bar{M}_{d}=[(d+2) \pi / d] \Gamma(d / 2+1)^{-2 / d} M_{d}$ (in particular $\bar{M}_{2}=\pi / 16$. In general $\bar{M}_{d}<1$, thus the Geisinger-Weidl bound (2.27) falls short of the conjectured expression of Harrell and Hermi.

\subsection{One cannot hear the shape of a drum}

In the quoted paper of Mark Kac [62] he says that he personally believed that one cannot hear the shape of a drum. A couple of years before Mark Kac' article, John Milnor [77], had constructed two non-congruent sixteen dimensional tori whose Laplace-Beltrami operators have exactly the same eigenvalues. In 1985 Toshikazu Sunada [94], then at Nagoya University in Japan, developed an algebraic framework that provided a new, systematic approach of considering Mark Kac's question. Using Sunada's technique several mathematicians constructed isospectral manifolds (e.g., Gordon and Wilson; Brooks; Buser, etc.). See, e.g., the review article of Robert Brooks (1988) with the situation on isospectrality up to that date in [36]. Finally, in 1992, Carolyn Gordon, David Webb and Scott Wolpert [54] gave the definite negative answer to Mark Kac's question and constructed two plane domains (henceforth called the GWW domains) with the same Dirichlet eigenvalues.

\subsection{Proof of isospectrality using transplantation}

The most elementary proof of isospectrality of the GWW domains is done using the method of transplantation. For the method of transplantation see, e.g., [27,28]. See also the expository article [29] by the same author. The method also appears briefly described in the article of Sridhar and Kudrolli cited in the Bibliographical Remarks, (iii) at the end of this chapter.

To conclude this chapter we will give the details of the proof of isospectrality of the GWW domains using transplantation (for the interested reader, there is a recent review article [53] that presents many different proofs of isospectrality, including transplantation, and paper folding techniques). For that purpose label from 1 to 7 the congruent triangles that make the two GWW domains (see Figure 1). Each of this isosceles right triangles has two cathets, labeled $A$ and $B$ and the hypothenuse, labeled $T$. Each of the pieces (triangles) that make each one of the two domains is connected to one or more neighboring triangles through a side $A$, a side $B$ or a side $T$. Each of the two isospectral domains has an associated graph, which are given in Figure 2. 
Fig. 1 GWW Isospectral Domains $D_{1}$ and $D_{2}$
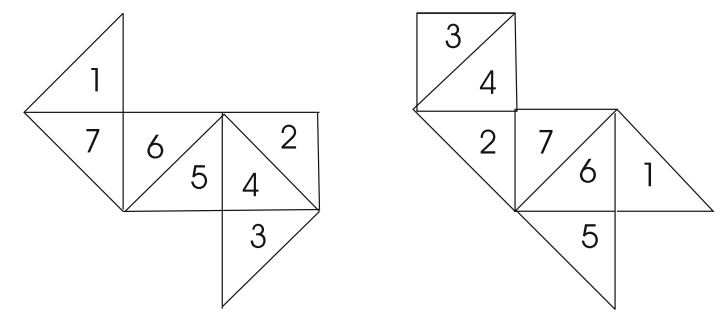

Fig. 2 Sunada Graphs corresponding to Domains $D_{1}$ and $D_{2}$
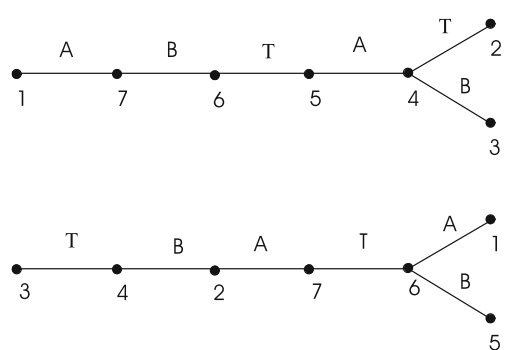

These graphs have their origin in the algebraic formulation of Sunada [94]. The vertices in each graph are labeled according to the number that each of the pieces (triangles) has in each of the given domains. As for the edges joining two vertices in these graphs, they are labeled by either an $A$, a $B$ or a $T$ depending on the type of the common side of two neighboring triangles in Figure 1. In order to show that both domains are isospectral it is convenient to consider any function defined on each domain as consisting of seven parts, each part being the restriction of the original function to each one of the individual triangles that make the domain. In this way, if $\psi$ is a function defined on the domain 1, we will write it as a vector with seven components, i.e., $\psi=\left[\psi_{i}\right]_{i=1}^{7}$, where $\psi_{i}$ is a scalar function whose support is triangle $i$ on the domain 1. Similarly, a function $\varphi$ defined over the domain 2 may be represented as a seven component vector $\varphi=\left[\varphi_{i}\right]_{i=1}^{7}$, with the equivalent meaning but referred to the second domain.

In order to show the isospectrality of the two domains we have to exhibit a mapping transforming the functions defined on the first domain into functions defined in the second domain. Given the decomposition we have made of the eigenfunctions as vectors of seven components, this transformation will be represented by a $7 \times 7$ matrix. In order to show that the two domains have the same spectra we need this matrix to be orthogonal. This matrix is given explicitly by

$$
T_{D}=\left(\begin{array}{ccccccc}
-a & a & a & -a & b & -b & b \\
a & -b & -a & b & -a & a & -b \\
a & -a & -b & b & -b & a & -a \\
-a & b & b & -a & a & -b & a \\
-b & a & b & -a & a & -a & b \\
b & -a & -a & b & -a & b & -a \\
-b & b & a & -a & b & -a & a
\end{array}\right) .
$$


For the matrix $T_{D}$ to be orthogonal, we need that the parameters $a$ and $b$ satisfy the following relations: $4 a^{2}+3 b^{2}=1,2 a^{2}+4 a b+b^{2}=0$, and $4 a+3 b=1$. Although we do not need the numerical values of $a$ and $b$ in the sequel, it is good to know that there is a solution to this system of equations, namely $a=(1-3 \sqrt{8} / 4) / 7$ and $b=(1+\sqrt{8}) / 7$. The matrix $T_{D}$ is orthogonal, i.e., $T_{D} T_{D}^{t}=1$. The label $D$ used here refers to the fact that this matrix $T_{D}$ is used to show isospectrality for the Dirichlet problem. A similar matrix can be constructed to show isospectrality for the Neumann problem. In order to show isospectrality it is not sufficient to show that the matrix $T_{D}$ is orthogonal. It must fulfill two additional properties. On the one hand it should transform a function $\psi$ that satisfies the Dirichlet conditions in the first domain in a function $\varphi$ that satisfies Dirichlet boundary conditions on the second domain. Moreover, by elliptic regularity, since the functions $\psi$ and its image $\varphi$ satisfy the eigenvalue equation $-\Delta u=\lambda u$, they must be smooth (in fact they should be real analytic in the interior of the corresponding domains), and therefore they must be continuous at the adjacent edges connecting two neighboring triangles. Thus, while the function $\psi$ is continuous when crossing the common edges of neighboring triangles in the domain 1 , the function $\varphi$ should be continuous when crossing the common edges of neighboring triangles in the domain 2 . These two properties are responsible for the peculiar structure of $a$ 's and $b$ 's in the components of the matrix $T_{D}$.

To illustrate these facts, if the function $\varphi$ is an eigenfunction of the Dirichlet problem for the domain 2, it must satisfy, among others, the following properties,

$$
\varphi_{2}^{A}=\varphi_{7}^{A}
$$

and

$$
\varphi_{5}^{T}=0
$$

Here $\varphi_{2}$ denotes the second component of $\varphi$, i.e., the restriction of the function $\varphi$ to the second triangle in Domain 2 (see Figure 2). On the other hand, $\varphi_{2}^{A}$ denotes the restriction of $\varphi_{2}$ on the edge $A$ of triangle 2. Since on the domain 2 , the triangles 2 and 7 are glued through a cathet of type $A,(2.29)$ is precisely the condition that $\varphi$ has to be smooth in the interior of 2. On the other hand, $\varphi$ must be a solution of the Dirichlet problem for the domain 2 and as such it must satisfy zero boundary conditions. Since the hypothenuse $T$ of triangle 5 is part of the boundary of the domain $2, \varphi$ must vanish there. This is precisely the condition (2.30). Let us check, as an exercise that if $\psi$ is smooth and satisfies Dirichlet boundary conditions in the domain 1, its image $\varphi=T_{D} \psi$ satisfies (2.30) over the domain 2. We let as an exercise to the reader to check (2.29), and all the other conditions on "smoothness" and boundary condition of $\varphi$ (this is a long but straightforward task). From (2.28) we have that

$$
\varphi_{5}^{T}=-b \psi_{1}^{T}+a \psi_{2}^{T}+b \psi_{3}^{T}-a \psi_{4}^{T}+a \psi_{5}^{T}-a \psi_{6}^{T}+b \psi_{7}^{T} .
$$

Since all the sides of type $T$ of the pieces 1,3 and 7 in the domain 1 are part of the boundary of the domain (see Figure 1), $\psi_{1}^{T}=\psi_{3}^{T}=\psi_{7}^{T}=0$. On the other hand, since 2 and 4 are neighboring triangles in the domain 1 , glued through a side of type 
$T$, we have $\psi_{2}^{T}=\psi_{4}^{T}$. By the same reasoning we have $\psi_{5}^{T}=\psi_{6}^{T}$. Using these three conditions on (2.31) we obtain (2.30). All the other conditions can be verified in a similar way. Collecting all these facts, we conclude with

Theorem 2.1 (P. Bérard) The transformation $T_{D}$ given by (2.28) is an isometry from $L^{2}\left(D_{1}\right)$ into $L^{2}\left(D_{2}\right)$ (here $D_{1}$ and $D_{2}$ are the two domains of Figures 1 and 2$)$, which induces an isometry from $H_{0}^{1}\left(D_{1}\right)$ into $H_{0}^{1}\left(D_{2}\right)$, and therefore we have,

Theorem 2.2 (C. Gordon, D. Webb, S. Wolpert) The domains $D_{1}$ and $D_{2}$ of Figures 1 and 2 are isospectral.

Although the proof by transplantation is straightforward to follow, it does not shed light on the rich geometric, analytic and algebraic structure of the problem initiated by Mark Kac. For the interested reader it is recommendable to read the papers of Sunada [94] and of Gordon, Webb and Wolpert [54].

In the previous paragraphs we have seen how the answer to the original Kac's question is in general negative. However, if we are willing to require some analyticity of the domains, and certain symmetries, we can recover uniqueness of the domain once we know the spectrum. During the last decade there has been an important progress in this direction. In 2000, S. Zelditch, [108] proved that in two dimensions, simply connected domains with the symmetry of an ellipse are completely determined by either their Dirichlet or their Neumann spectrum. More recently [109], he proved a much stronger positive result. Consider the class of planar domains with no holes and very smooth boundary and with at least one mirror symmetry. Then one can recover the shape of the domain given the Dirichlet spectrum.

\subsection{Bibliographical remarks}

i) The sentence of Arthur Schuster (1851-1934) quoted at the beginning of this chapter is cited in Reed and Simon's book, volume IV [90]. It is taken from the article A. Schuster, The Genesis of Spectra, in Report of the fifty-second meeting of the British Association for the Advancement of Science (held at Southampton in August 1882), Brit. Assoc. Rept., pp. 120-121, 1883. Arthur Schuster was a British physicist (he was a leader spectroscopist at the turn of the XIX century). It is interesting to point out that Arthur Schuster found the solution to the Lane-Emden equation with exponent 5, i.e., to the equation,

$$
-\Delta u=u^{5}
$$

in $\mathbb{R}^{3}$, with $u>0$ going to zero at infinity. The solution is given by

$$
u=\frac{3^{1 / 4}}{\left(1+|x|^{2}\right)^{1 / 2}} .
$$

(A. Schuster, On the internal constitution of the Sun, Brit. Assoc. Rept. pp. 427-429, 1883). Since the Lane-Emden equation for exponent 5 is the Euler-Lagrange equation 
for the minimizer of the Sobolev quotient, this is precisely the function that, modulo translations and dilations, gives the best Sobolev constant. For a nice autobiography of Arthur Schuster see A. Schuster, Biographical fragments, Mc Millan \& Co., London, (1932).

ii) A very nice short biography of Marc Kac was written by H. P. McKean [Mark Kac in Bibliographical Memoirs, National Academy of Science, 59, 214-235 (1990); available on the web (page by page) at http://www.nap.edu/books/0309041988/html/ 214.html]. The reader may want to read his own autobiography: Mark Kac, Enigmas of Chance, Harper and Row, NY, 1985 [reprinted in 1987 in paperback by The University of California Press]. For his article in the American Mathematical Monthly, op. cit., Mark Kac obtained the 1968 Chauvenet Prize of the Mathematical Association of America.

iii) For a beautiful account of the scientific contributions of Lipman Bers (1993-1914), who coined the famous phrase, Can one hear the shape of a drum?, see the article by Cathleen Morawetz and others, Remembering Lipman Bers, Notices of the AMS 42, 8-25 (1995).

iv) It is interesting to remark that the values of the first Dirichlet eigenvalues of the GWW domains were obtained experimentally by S. Sridhar and A. Kudrolli [93], Experiments on Not "Hearing the Shape" of Drums, Physical Review Letters, 72, 2175-2178 (1994). In that article one can find the details of the physics experiments performed by these authors using very thin electromagnetic resonant cavities with the shape of the Gordon-Webb-Wolpert (GWW) domains. This is the first time that the approximate numerical values of the first 25 eigenvalues of the two GWW were obtained. The corresponding eigenfunctions are also displayed. A quick reference to the transplantation method of Pierre Berard is also given in this article, including the transplantation matrix connecting the two isospectral domains. The reader may want to check the web page of S. Sridhar's Lab (http://sagar.physics.neu.edu/) for further experiments on resonating cavities, their eigenvalues and eigenfunctions, as well as on experiments on quantum chaos.

v) The numerical computation of the eigenvalues and eigenfunctions of the pair of GWW isospectral domains was obtained by Tobin A. Driscoll, Eigenmodes of isospectral domains, SIAM Review 39, 1-17 (1997).

vi) In its simplified form, the Gordon-Webb-Wolpert domains (GWW domains) are made of seven congruent rectangle isosceles triangles. Certainly the GWW domains have the same area, perimeter and connectivity. The GWW domains are not convex. Hence, one may still ask the question whether one can hear the shape of a convex drum. There are examples of convex isospectral domains in higher dimension (see e.g. C. Gordon and D. Webb, Isospectral convex domains in Euclidean Spaces, Math. Res. Letts. 1, 539-545 (1994), where they construct convex isospectral domains in $\left.\mathbb{R}^{n}, n \geq 4\right)$. Remark: For an update of the Sunada Method, and its applications see the article of Robert Brooks [The Sunada Method, in Tel Aviv Topology Conference "Rothenberg Festschrift" 1998, Contemporary Mathematics 231, 25-35 (1999); electronically available at: http://www.math.technion.ac.il/ rbrooks].

vii) There is a vast literature on Kac's question, and many review lectures on it. In particular, this problem belongs to a very important branch of mathematics: Inverse 
Problems. In that connection, see the lectures of R. Melrose [76]. For a very recent review on Kac's question and its many ramifications in physics, see [53].

\section{Rearrangements}

\subsection{Definition and basic properties}

For many problems of functional analysis it is useful to replace some function by an equimeasurable but more symmetric one. This method, which was first introduced by Hardy and Littlewood, is called rearrangement or Schwarz symmetrization [55]. Among several other applications, it plays an important role in the proofs of isoperimetric inequalities like the Rayleigh-Faber-Krahn inequality, the Szegö-Weinberger inequality or the Payne-Pólya-Weinberger inequality (see Sections 4, 5 and 6 below). In the following we present some basic definitions and theorems concerning spherically symmetric rearrangements.

We let $\Omega$ be a measurable subset of $\mathbb{R}^{n}$ and write $|\Omega|$ for its Lebesgue measure, which may be finite or infinite. If it is finite we write $\Omega^{\star}$ for an open ball with the same measure as $\Omega$, otherwise we set $\Omega^{\star}=\mathbb{R}^{n}$. We consider a measurable function $u: \Omega \rightarrow \mathbb{R}$ and assume either that $|\Omega|$ is finite or that $u$ decays at infinity, i.e., $|\{x \in \Omega:|u(x)|>t\}|$ is finite for every $t>0$.

Definition 3.1 The function

$$
\mu(t)=|\{x \in \Omega:|u(x)|>t\}|, \quad t \geq 0
$$

is called distribution function of $u$.

From this definition it is straightforward to check that $\mu(t)$ is a decreasing (nonincreasing), right-continuous function on $\mathbb{R}^{+}$with $\mu(0)=|\operatorname{sprt} u|$ and sprt $\mu=$ $[0$, ess sup $|u|)$.

\section{Definition 3.2}

- The decreasing rearrangement $u^{\sharp}: \mathbb{R}^{+} \rightarrow \mathbb{R}^{+}$of $u$ is the distribution function of $\mu$.

- The symmetric decreasing rearrangement $u^{\star}: \Omega^{\star} \rightarrow \mathbb{R}^{+}$of $u$ is defined by $u^{\star}(x)=$ $u^{\sharp}\left(C_{n}|x|^{n}\right)$, where $C_{n}=\pi^{n / 2}[\Gamma(n / 2+1)]^{-1}$ is the measure of the $n$-dimensional unit ball.

Because $\mu$ is a decreasing function, Definition 3.2 implies that $u^{\sharp}$ is an essentially inverse function of $\mu$. The names for $u^{\sharp}$ and $u^{\star}$ are justified by the following two lemmas:

\section{Lemma 3.3}

(a) The function $u^{\sharp}$ is decreasing, $u^{\sharp}(0)=\operatorname{esssup}|u|$ and $\operatorname{sprt} u^{\sharp}=[0,|\operatorname{sprt} u|)$

(b) $u^{\sharp}(s)=\min \{t \geq 0: \mu(t) \leq s\}$

(c) $u^{\sharp}(s)=\int_{0}^{\infty} \chi_{[0, \mu(t))}(s) \mathrm{d} t$ 
(d) $\left|\left\{s \geq 0: u^{\sharp}(s)>t\right\}\right|=|\{x \in \Omega:|u(x)|>t\}|$ for all $t \geq 0$.

(e) $\left\{s \geq 0: u^{\sharp}(s)>t\right\}=[0, \mu(t))$ for all $t \geq 0$.

Proof Part (a) is a direct consequence of the definition of $u^{\sharp}$, keeping in mind the general properties of distribution functions stated above. The representation formula in part (b) follows from

$u^{\sharp}(s)=|\{w \geq 0: \mu(w)>s\}|=\sup \{w \geq 0: \mu(w)>s\}=\min \{w \geq 0: \mu(w) \leq s\}$,

where we have used the definition of $u^{\sharp}$ in the first step and then the monotonicity and right-continuity of $\mu$. Part (c) is a consequence of the 'layer-cake formula', see Theorem 10.1 in the appendix. To prove part (d) we need to show that

$$
\left\{s \geq 0: u^{\sharp}(s)>t\right\}=[0, \mu(t)) .
$$

Indeed, if $s$ is an element of the left hand side of (3.1), then by Lemma 3.3, part (b), we have

$$
\min \{w \geq 0: \mu(w) \leq s\}>t
$$

But this means that $\mu(t)>s$, i.e., $s \in[0, \mu(t))$. On the other hand, if $s$ is an element of the right hand side of (3.1), then $s<\mu(t)$ which implies again by part (b) that

$$
u^{\sharp}(s)=\min \{w \geq 0: \mu(w) \leq s\} \geq \min \{w \geq 0: \mu(w)<\mu(t)\}>t,
$$

i.e., $s$ is also an element of the left hand side. Finally, part (e) is a direct consequence of part (d).

It is straightforward to transfer the statements of Lemma 3.3 to the symmetric decreasing rearrangement:

\section{Lemma 3.4}

(a) The function $u^{\star}$ is spherically symmetric and radially decreasing.

(b) The measure of the level set $\left\{x \in \Omega^{\star}: u^{\star}(x)>t\right\}$ is the same as the measure of $\{x \in \Omega:|u(x)|>t\}$ for any $t \geq 0$ (Figure 3 ).

From Lemma 3.3 (c) and Lemma 3.4 (b) we see that the three functions $u, u^{\sharp}$ and $u^{\star}$ have the same distribution function and therefore they are said to be equimeasurable. Quite analogous to the decreasing rearrangements one can also define increasing ones:

\section{Definition 3.5}

- If the measure of $\Omega$ is finite, we call $u_{\sharp}(s)=u^{\sharp}(|\Omega|-s)$ the increasing rearrangement of $u$.

- The symmetric increasing rearrangement $u_{\star}: \Omega^{\star} \rightarrow \mathbb{R}^{+}$of $u$ is defined by $u_{\star}(x)=$ $u_{\sharp}\left(C_{n}|x|^{n}\right)$. 

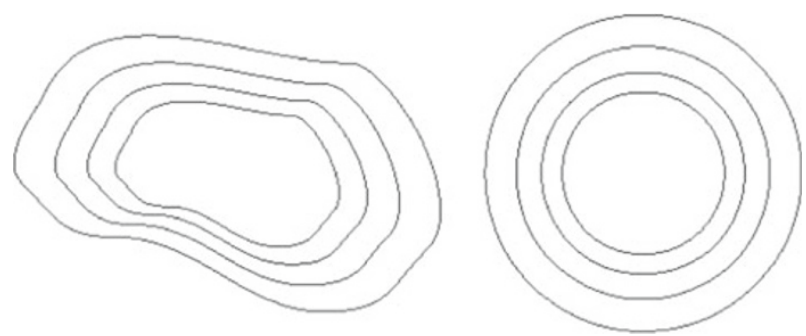

Fig. 3 The level sets of the functions $u$ (left) and of its rearrangement $u^{*}$ (right)

In his lecture notes on rearrangements (see the reference in the Bibliographical Remarks, i) at the end of this chapter), G. Talenti, gives the following example, illustrating the meaning of the distribution and the rearrangement of a function: Consider the function $u(x) \equiv 8+2 x^{2}-x^{4}$, defined on the interval $-2 \leq x \leq 2$. Then, it is a simple exercise to check that the corresponding distribution function $\mu(t)$ is given by

$$
\mu(t)= \begin{cases}2 \sqrt{1+\sqrt{9-t}} & \text { if } 0 \leq t \leq 8, \\ 2 \sqrt{2-2 \sqrt{t-8}} & \text { if } 8<t \leq 9 .\end{cases}
$$

Hence,

$$
u^{\star}(x)= \begin{cases}9-x^{2}+x^{4} / 4 & \text { if } x \leq \sqrt{2} \\ u(x) & \text { if }|x|>\sqrt{2} .\end{cases}
$$

This function can as well be used to illustrate the theorems below.

\subsection{Main theorems}

Rearrangements are a useful tool of functional analysis because they considerably simplify a function without changing certain properties or at least changing them in a controllable way. The simplest example is the fact that the integral of a function's absolute value is invariant under rearrangement. A bit more generally, we have:

Theorem 3.6 Let $\Phi$ be a continuous increasing map from $\mathbb{R}^{+}$to $\mathbb{R}^{+}$with $\Phi(0)=0$. Then

$$
\int_{\Omega^{\star}} \Phi\left(u^{\star}(x)\right) \mathrm{d} x=\int_{\Omega} \Phi(|u(x)|) \mathrm{d} x=\int_{\Omega^{\star}} \Phi\left(u_{\star}(x)\right) \mathrm{d} x .
$$

Proof The theorem follows directly from Theorem 10.1 in the appendix: If we choose $m(\mathrm{~d} x)=\mathrm{d} x$, the right hand side of (10.1) takes the same value for $v=|u|, v=u^{\star}$ and $v=u_{\star}$. The conditions on $\Phi$ are necessary since $\Phi(t)=v([0, t))$ must hold for some measure $v$ on $\mathbb{R}^{+}$. 
For later reference we state a rather specialized theorem, which is an estimate on the rearrangement of a spherically symmetric function that is defined on an asymmetric domain:

Theorem 3.7 Assume that $u_{\Omega}: \Omega \rightarrow \mathbb{R}^{+}$is given by $u_{\Omega}(x)=u(|x|)$, where $u$ : $\mathbb{R}^{+} \rightarrow \mathbb{R}^{+}$is a non-negative decreasing (resp. increasing) function. Then $u_{\Omega}^{\star}(x) \leq$ $u(|x|)\left(\right.$ resp. $\left.u_{\Omega \star}(x) \geq u(|x|)\right)$ for every $x \in \Omega^{\star}$.

Proof Assume first that $u$ is a decreasing function. The layer-cake representation for $u_{\Omega}^{\star}$ is

$$
\begin{aligned}
u_{\Omega}^{\star}(x)=u^{\sharp}\left(C_{n}|x|^{n}\right) & =\int_{0}^{\infty} \chi_{\left[0,\left|\left\{x \in \Omega: u_{\Omega}(x)>t\right\}\right|\right)}\left(C_{n}|x|^{n}\right) \mathrm{d} t \\
& \leq \int_{0}^{\infty} \chi_{\left[0,\left|\left\{x \in \mathbb{R}^{n}: u(|x|)>t\right\}\right|\right)}\left(C_{n}|x|^{n}\right) \mathrm{d} t \\
& =\int_{0}^{\infty} \chi_{\left\{x \in \mathbb{R}^{n}: u(|x|)>t\right\}}(x) \mathrm{d} t \\
& =u(|x|) .
\end{aligned}
$$

The product of two functions changes in a controllable way under rearrangement:

Theorem 3.8 Suppose that $u$ and $v$ are measurable and non-negative functions defined on some $\Omega \subset \mathbb{R}^{n}$ with finite measure. Then

$$
\int_{\mathbb{R}^{+}} u^{\sharp}(s) v^{\sharp}(s) \mathrm{d} s \geq \int_{\Omega} u(x) v(x) \mathrm{d} x \geq \int_{\mathbb{R}^{+}} u^{\sharp}(s) v_{\sharp}(s) \mathrm{d} s
$$

and

$$
\int_{\Omega^{\star}} u^{\star}(x) v^{\star}(x) \mathrm{d} x \geq \int_{\Omega} u(x) v(x) \mathrm{d} x \geq \int_{\Omega^{\star}} u^{\star}(x) v_{\star}(x) \mathrm{d} x .
$$

Proof We first show that for every measurable $\Omega^{\prime} \subset \Omega$ and every measurable $v$ : $\Omega \rightarrow \mathbb{R}^{+}$the relation

$$
\int_{0}^{\left|\Omega^{\prime}\right|} v^{\sharp}(s) \mathrm{d} s \geq \int_{\Omega^{\prime}} v \mathrm{~d} x \geq \int_{0}^{\left|\Omega^{\prime}\right|} v_{\sharp}(s) \mathrm{d} s
$$

holds: We can assume without loss of generality that $v$ is integrable. Then the layercake formula (see Theorem 10.1 in the appendix) gives

$$
v=\int_{0}^{\infty} \chi_{\{x \in \Omega: v(x)>t\}} \mathrm{d} t \quad \text { and } \quad v^{\sharp}=\int_{0}^{\infty} \chi_{[0, \mu(t))} \mathrm{d} t .
$$


Hence,

$$
\begin{gathered}
\int_{\Omega^{\prime}} v \mathrm{~d} x=\int_{0}^{\infty}\left|\Omega^{\prime} \cap\{x \in \Omega: v(x)>t\}\right| \mathrm{d} t, \\
\int_{0}^{\left|\Omega^{\prime}\right|} v^{\sharp}(s) \mathrm{d} s=\int_{0}^{\infty} \min \left(\left|\Omega^{\prime}\right|,|\{x \in \Omega: v(x)>t\}|\right) \mathrm{d} t .
\end{gathered}
$$

The first inequality in (3.4) follows. The second inequality in (3.4) can be established with the help of the first:

$$
\begin{aligned}
\int_{0}^{\left|\Omega^{\prime}\right|} v_{\sharp} \mathrm{d} s & =\int_{0}^{|\Omega|} v_{\sharp} \mathrm{d} s-\int_{\left|\Omega^{\prime}\right|}^{|\Omega|} v_{\sharp} \mathrm{d} s=\int_{\Omega} v \mathrm{~d} x-\int_{0}^{|\Omega|-\left|\Omega^{\prime}\right|} v^{\sharp} \mathrm{d} s \\
& \leq \int_{\Omega} v \mathrm{~d} x-\int_{\Omega \backslash \Omega^{\prime}} v \mathrm{~d} x=\int_{\Omega^{\prime}} v \mathrm{~d} x .
\end{aligned}
$$

Now assume that $u$ and $v$ are measurable, non-negative and-without loosing generality-integrable. Since we can replace $v$ by $u$ in the equations (3.5), we have

$$
\begin{aligned}
\int_{\Omega} u(x) v(x) \mathrm{d} x & =\int_{0}^{\infty} \mathrm{d} t \int_{\{x \in \Omega: u(x)>t\}} v(x) \mathrm{d} x, \\
\int_{0}^{\infty} u^{\sharp}(s) v^{\sharp}(s) \mathrm{d} s & =\int_{0}^{\infty} \mathrm{d} t \int_{0}^{\mu(t)} v^{\sharp}(s) \mathrm{d} s,
\end{aligned}
$$

where $\mu$ is the distribution function of $u$. On the other hand, the first inequality in (3.4) tells us that

$$
\int_{\{x \in \Omega: u(x)>t\}} v(x) \mathrm{d} x \leq \int_{0}^{\mu(t)} v^{\sharp}(s) \mathrm{d} s
$$

for every non-negative $t$, such that the first inequality in (3.2) follows. The second part of (3.2) can be proven completely analogously, and the inequalities (3.3) are a direct consequence of (3.2).

\subsection{Gradient estimates}

The integral of a function's gradient over the boundary of a level set can be estimated in terms of the distribution function:

Theorem 3.9 Assume that $u: \mathbb{R}^{n} \rightarrow \mathbb{R}$ is Lipschitz continuous and decays at infinity, i.e., the measure of $\Omega_{t}:=\left\{x \in \mathbb{R}^{n}:|u(x)|>t\right\}$ is finite for every positive $t$. If $\mu$ is the distribution function of $u$ then

$$
\int_{\partial \Omega_{t}}|\nabla u| H_{n-1}(\mathrm{~d} x) \geq-n^{2} C_{n}^{2 / n} \frac{\mu(t)^{2-2 / n}}{\mu^{\prime}(t)} .
$$


Remarks i) Here $H_{n}(A)$ denotes the $n$-dimensional Hausdorff measure of the set $A$ (see, e.g., [51]); ii) it is worth to point out that (3.6) is an equality for radial functions.

Proof On the one hand, by the classical isoperimetric inequality we have

$$
\int_{\partial \Omega_{t}} H_{n-1}(\mathrm{~d} x) \geq n C_{n}^{1 / n}\left|\Omega_{t}\right|^{1-1 / n}=n C_{n}^{1 / n} \mu(t)^{1-1 / n} .
$$

On the other hand, we can use the Cauchy-Schwarz inequality to get

$$
\begin{aligned}
\int_{\partial \Omega_{t}} H_{n-1}(\mathrm{~d} x) & =\int_{\partial \Omega_{t}} \frac{\sqrt{|\nabla u|}}{\sqrt{|\nabla u|}} H_{n-1}(\mathrm{~d} x) \\
& \leq\left(\int_{\partial \Omega_{t}}|\nabla u| H_{n-1}(\mathrm{~d} x)\right)^{1 / 2}\left(\int_{\partial \Omega_{t}} \frac{1}{|\nabla u|} H_{n-1}(\mathrm{~d} x)\right)^{1 / 2} .
\end{aligned}
$$

The last integral in the above formula can be replaced by $-\mu^{\prime}(t)$ according to Federer's coarea formula (see, [51]). The result is

$$
\int_{\partial \Omega_{t}} H_{n-1}(\mathrm{~d} x) \leq\left(\int_{\partial \Omega_{t}}|\nabla u| H_{n-1}(\mathrm{~d} x)\right)^{1 / 2}\left(-\mu^{\prime}(t)\right)^{1 / 2} .
$$

Comparing the equations (3.7) and (3.8) yields Theorem 3.9.

Integrals that involve the norm of the gradient can be estimated using the following important theorem:

Theorem 3.10 Let $\Phi: \mathbb{R}^{+} \rightarrow \mathbb{R}^{+}$be a Young function, i.e., $\Phi$ is increasing and convex with $\Phi(0)=0$. Suppose that $u: \mathbb{R}^{n} \rightarrow \mathbb{R}$ is Lipschitz continuous and decays at infinity. Then

$$
\int_{\mathbb{R}^{n}} \Phi\left(\left|\nabla u^{\star}(x)\right|\right) \mathrm{d} x \leq \int_{\mathbb{R}^{n}} \Phi(|\nabla u(x)|) \mathrm{d} x .
$$

For the special case $\Phi(t)=t^{2}$ Theorem 3.10 states that the "energy expectation value' of a function decreases under symmetric rearrangement, a fact that is key to the proof of the Rayleigh-Faber-Krahn inequality (see Section 4.1).

Proof Theorem 3.10 is a consequence of the following chain of (in)equalities, the second step of which follows from Lemma 3.11 below.

$$
\begin{aligned}
\int_{\mathbb{R}^{n}} \Phi(|\nabla u|) \mathrm{d} x & =\int_{0}^{\infty} \mathrm{d} s \frac{\mathrm{d}}{\mathrm{d} s} \int_{\left\{x \in \mathbb{R}^{n}:|u(x)|>u^{*}(s)\right\}} \Phi(|\nabla u|) \mathrm{d} x \\
& \geq \int_{0}^{\infty} \mathrm{d} s \Phi\left(-n C_{n}^{1 / n} s^{1-1 / n} \frac{\mathrm{d} u^{*}}{\mathrm{~d} s}(s)\right)=\int_{\mathbb{R}^{n}} \Phi\left(\left|\nabla u^{\star}\right|\right) \mathrm{d} x .
\end{aligned}
$$


Lemma 3.11 Let $u$ and $\Phi$ be as in Theorem 3.10. Then for almost every positive $s$ holds

$$
\frac{\mathrm{d}}{\mathrm{d} s} \int_{\left\{x \in \mathbb{R}^{n}:|u(x)|>u^{*}(s)\right\}} \Phi(|\nabla u|) \mathrm{d} x \geq \Phi\left(-n C_{n}^{1 / n} s^{1-1 / n} \frac{\mathrm{d} u^{*}}{\mathrm{~d} s}(s)\right) .
$$

Proof First we prove Lemma 3.11 for the special case of $\Phi$ being the identity. If $s>|\operatorname{sprt} u|$ then (3.9) is clearly true since both sides vanish. Thus we can assume that $0<s<|\operatorname{sprt} u|$. For all $0 \leq a<b<|\operatorname{sprt} u|$ we show that

$$
\int_{\left\{x \in \mathbb{R}^{n}: u^{*}(a)>|u(x)|>u^{*}(b)\right\}}|\nabla u(x)| \mathrm{d} x \geq n C_{n}^{1 / n} a^{1-1 / n}\left(u^{*}(a)-u^{*}(b)\right) .
$$

The statement (3.10) is proven by the following chain of inequalities, in which we first use Federer's coarea formula, then the classical isoperimetric inequality in $\mathbb{R}^{n}$ and finally the monotonicity of the integrand:

$$
\begin{aligned}
\text { 1.h.s. of (3.10) } & =\int_{u^{*}(b)}^{u^{*}(a)} H_{n-1}\left\{x \in \mathbb{R}^{n}:|u(x)|=t\right\} \mathrm{d} t \\
& \geq \int_{u^{*}(b)}^{u^{*}(a)} n C_{n}^{1 / n}\left|\left\{x \in \mathbb{R}^{n}:|u(x)| \geq t\right\}\right|^{1-1 / n} \mathrm{~d} t \\
& \geq n C_{n}^{1 / n}\left|\left\{x \in \mathbb{R}^{n}:|u(x)| \geq u^{*}(a)\right\}\right|^{1-1 / n} \cdot\left(u^{*}(a)-u^{*}(b)\right) \\
& \geq \text { r.h.s. of }(3.10) .
\end{aligned}
$$

In the case of $\Phi$ being the identity, Lemma 3.11 follows from (3.10): Replace $b$ by $a+\epsilon$ with some $\epsilon>0$, multiply both sides by $\epsilon^{-1}$ and then let $\epsilon$ go to zero.

It remains to show that equation (3.9) holds for almost every $s>0$ if $\Phi$ is not the identity but some general Young function. From the monotonicity of $u^{*}$ follows that for almost every $s>0$ either $\frac{\mathrm{d} u^{*}}{\mathrm{~d} s}$ is zero or there is a neighborhood of $s$ where $u^{*}$ is continuous and decreases strictly. In the first case there is nothing to prove, thus we can assume the second one. Then we have

$$
\left|\left\{x \in \mathbb{R}^{n}: u^{*}(s) \geq|u(x)|>u^{*}(s+\epsilon)\right\}\right|=\epsilon
$$

for small enough $\epsilon>0$. Consequently, we can apply Jensen's inequality to get

$$
\begin{aligned}
& \frac{1}{\epsilon} \int_{\left\{x \in \mathbb{R}^{n}: u^{*}(s) \geq|u(x)|>u *(s+h)\right\}} \Phi(|\nabla u(x)|) \mathrm{d} x \\
& \geq \Phi\left(\frac{1}{\epsilon} \int_{\left\{x \in \mathbb{R}^{n}: u^{*}(s) \geq|u(x)|>u *(s+h)\right\}}|\nabla u(x)| \mathrm{d} x\right) .
\end{aligned}
$$


Taking the limit $\epsilon \downarrow 0$, this yields

$$
\frac{\mathrm{d}}{\mathrm{d} s} \int_{\left\{x \in \mathbb{R}^{n}:|u(x)|>u *(s)\right\}} \Phi(|\nabla u(x)|) \mathrm{d} x \geq \Phi\left(\frac{\mathrm{d}}{\mathrm{d} s} \int_{\left\{x \in \mathbb{R}^{n}:|u(x)|>u *(s)\right\}}|\nabla u(x)| \mathrm{d} x\right) .
$$

Since we have already proven Lemma 3.11 for the case of $\Phi$ being the identity, we can apply it to the argument of $\Phi$ on the right hand side of the above inequality. The statement of Lemma 3.11 for general $\Phi$ follows.

\subsection{Bibliographical remarks}

i) Rearrangements of functions were introduced by G. Hardy and J. E. Littlewood. Their results are contained in the classical book of G.H. Hardy, J. E. Littlewood, and G. Pólya [55]. The fact that the $L^{2}$ norm of the gradient of a function decreases under rearrangements was proven by Faber and Krahn (see references below). A more modern proof as well as many results on rearrangements and their applications to PDE's can be found in [100]. See also Chapter 3, pp. 79-ff in reference [69]. The reader may want to see also the article by E.H. Lieb, Existence and uniqueness of the minimizing solution of Choquard's nonlinear equation, Studies in Appl. Math. 57, 93-105 (1976/77), for an alternative proof of the fact that the $L^{2}$ norm of the gradient decreases under rearrangements using heat kernel techniques. An excellent expository review on rearrangements of functions (with a good bibliography) can be found in G. Talenti, Inequalities in rearrangement invariant function spaces, in Nonlinear analysis, function spaces and applications, Vol. 5 (Prague, 1994), 177-230, Prometheus, Prague, 1994. (available at the website: http://www.emis.de/proceedings/Praha94/). The Riesz rearrangement inequality is the assertion that for nonnegative measurable functions $f, g, h$ in $\mathbb{R}^{n}$, we have

$$
\int_{\mathbb{R}^{n} \times \mathbb{R}^{n}} f(y) g(x-y) h(x) d x d y \leq \int_{\mathbb{R}^{n} \times \mathbb{R}^{n}} f^{\star}(y) g^{\star}(x-y) h^{\star}(x) d x d y .
$$

For $n=1$ the inequality is due to F. Riesz, Sur une inégalité intégrale, Journal of the London Mathematical Society 5, 162-168 (1930). For general $n$ is due to S.L. Sobolev, On a theorem of functional analysis, Mat. Sb. (NS) 4, 471-497 (1938) [the English translation appears in AMS Translations (2) 34, 39-68 (1963)]. The cases of equality in the Riesz inequality were studied by A. Burchard, Cases of equality in the Riesz rearrangement inequality, Annals of Mathematics 143 499-627 (1996) (this paper also has an interesting history of the problem). In addition to Burchard's thorough analysis, there is a strictness statement in Lieb's paper on the Choquard's nonlinear equation, cited above, which is useful in applications.

ii) Rearrangements of functions have been extensively used to prove symmetry properties of positive solutions of nonlinear PDE's. See, e.g., B. Kawohl, Rearrangements and convexity of level sets in Partial Differential Equations, Lecture Notes in Mathematics, 1150, Springer-Verlag, Berlin (1985), and references therein. 
iii) There are different types of rearrangements of functions. See, e.g., the review article of Al Baernstein [19]. For an interesting approach to rearrangements see also [35]. This approach goes back through Baernstein-Taylor (A. Baernstein and B. A. Taylor, Spherical rearrangements, subharmonic functions and $*$-functions in $n$-space, Duke Math. J. 43, 245-268 (1976)), who cite Ahlfors (L. V. Ahlfors, Conformal Invariants, McGraw Hill, NY, 1973), who in turn credits Hardy and Littlewood.

\section{The Rayleigh-Faber-Krahn inequality}

\subsection{The Euclidean case}

Many isoperimetric inequalities have been inspired by the question which geometrical layout of some physical system maximizes or minimizes a certain quantity. One may ask, for example, how matter of a given mass density must be distributed to minimize its gravitational energy, or which shape a conducting object must have to maximize its electrostatic capacity. The most famous question of this kind was put forward at the end of the $19^{\text {th }}$ century by Lord Rayleigh in his work on the theory of sound [88]: he conjectured that among all drums of the same area and the same tension the circular drum produces the lowest fundamental frequency. This statement was proven independently in the 1920s by Faber [50] and Krahn [64,65].

To treat the problem mathematically, we consider an open bounded domain $\Omega \subset \mathbb{R}^{2}$ which matches the shape of the drum. Then the oscillation frequencies of the drum are given by the eigenvalues of the Laplace operator $-\Delta_{D}^{\Omega}$ on $\Omega$ with Dirichlet boundary conditions, up to a constant that depends on the drum's tension and mass density. In the following we will allow the more general case $\Omega \subset \mathbb{R}^{n}$ for $n \geq 2$, although the physical interpretation as a drum only makes sense if $n=2$. We define the Laplacian $-\Delta_{D}^{\Omega}$ via the quadratic-form approach, i.e., it is the unique self-adjoint operator in $L^{2}(\Omega)$ which is associated with the closed quadratic form

$$
h[\Psi]=\int_{\Omega}|\nabla \Psi|^{2} \mathrm{~d} x, \quad \Psi \in H_{0}^{1}(\Omega) .
$$

Here $H_{0}^{1}(\Omega)$, which is a subset of the Sobolev space $W^{1,2}(\Omega)$, is the closure of $C_{0}^{\infty}(\Omega)$ with respect to the form norm

$$
|\cdot|_{h}^{2}=h[\cdot]+\|\cdot\|_{L^{2}(\Omega)} \cdot
$$

For more details about the important question of how to define the Laplace operator on arbitrary domains and subject to different boundary conditions we refer the reader to $[46,32]$.

The spectrum of $-\Delta_{D}^{\Omega}$ is purely discrete since $H_{0}^{1}(\Omega)$ is, by Rellich's theorem, compactly imbedded in $L^{2}(\Omega)$ (see, e.g., [32]). We write $\lambda_{1}(\Omega)$ for the lowest eigenvalue of $-\Delta_{D}^{\Omega}$.

Theorem 4.1 (Rayleigh-Faber-Krahn inequality) Let $\Omega \subset \mathbb{R}^{n}$ be an open bounded domain with smooth boundary and $\Omega^{\star} \subset \mathbb{R}^{n}$ a ball with the same measure as $\Omega$. Then, 


$$
\lambda_{1}\left(\Omega^{*}\right) \leq \lambda_{1}(\Omega)
$$

with equality if and only if $\Omega$ itself is a ball.

Proof With the powerful mathematical tool of rearrangements (see Chapter 3) at hand, the proof of the Rayleigh-Faber-Krahn inequality is actually not difficult. Let $\Psi$ be the positive normalized first eigenfunction of $-\Delta_{D}^{\Omega}$. Since the domain of a positive self-adjoint operator is a subset of its form domain, we have $\Psi \in H_{0}^{1}(\Omega)$. Then we have $\Psi^{\star} \in H_{0}^{1}\left(\Omega^{\star}\right)$. Thus we can apply first the min-max principle and then the Theorems 3.6 and 3.10 to obtain

$$
\lambda_{1}\left(\Omega^{\star}\right) \leq \frac{\int_{\Omega^{\star}}\left|\nabla \Psi^{\star}\right|^{2} \mathrm{~d}^{n} x}{\int_{\Omega^{\star}}\left|\Psi^{*}\right|^{2} \mathrm{~d}^{n} x} \leq \frac{\int_{\Omega}|\nabla \Psi|^{2} \mathrm{~d}^{n} x}{\int_{\Omega} \Psi^{2} \mathrm{~d}^{n} x}=\lambda_{1}(\Omega) .
$$

The Rayleigh-Faber-Krahn inequality has been extended to a number of different settings, for example to Laplace operators on curved manifolds or with respect to different measures. In the following we shall give an overview of these generalizations.

\subsection{Schrödinger operators}

It is not difficult to extend the Rayleigh-Faber-Krahn inequality to Schrödinger operators, i.e., to operators of the form $-\Delta+V(x)$. Let $\Omega \subset \mathbb{R}^{n}$ be an open bounded domain and $V: \mathbb{R}^{n} \rightarrow \mathbb{R}^{+}$a non-negative potential in $L^{1}(\Omega)$. Then the quadratic form

$$
h_{V}[u]=\int_{\Omega}\left(|\nabla u|^{2}+V(x)|u|^{2}\right) \mathrm{d}^{n} x
$$

defined on

$$
\operatorname{Dom} h_{V}=H_{0}^{1}(\Omega) \cap\left\{u \in L^{2}(\Omega): \int_{\Omega}(1+V(x))|u(x)|^{2} \mathrm{~d}^{n} x<\infty\right\}
$$

is closed (see, e.g., $[45,46])$. It is associated with the positive self-adjoint Schrödinger operator $H_{V}=-\Delta+V(x)$. The spectrum of $H_{V}$ is purely discrete and we write $\lambda_{1}(\Omega, V)$ for its lowest eigenvalue.

Theorem 4.2 Under the assumptions stated above,

$$
\lambda_{1}\left(\Omega^{*}, V_{\star}\right) \leq \lambda_{1}(\Omega, V) .
$$

Proof Let $u_{1} \in$ Dom $h_{V}$ be the positive normalized first eigenfunction of $H_{V}$. Then we have $u_{1}^{\star} \in H_{0}^{1}\left(\Omega^{\star}\right)$ and by Theorem 3.8

$$
\int_{\Omega^{\star}}\left(1+V_{\star}\right) u_{1}^{\star 2} \mathrm{~d}^{n} x \leq \int_{\Omega}(1+V) u_{1}^{2} \mathrm{~d}^{n} x<\infty .
$$


Thus $u_{1}^{\star} \in$ Dom $h_{V_{\star}}$ and we can apply first the min-max principle and then Theorems $3.6,3.8$ and 3.10 to obtain

$$
\begin{aligned}
\lambda_{1}\left(\Omega^{\star}, V_{\star}\right) & \leq \frac{\int_{\Omega^{\star}}\left(\left|\nabla u_{1}^{\star}\right|^{2}+V_{\star} u_{1}^{\star 2}\right) \mathrm{d}^{n} x}{\int_{\Omega^{\star}}\left|u_{1}^{\star}\right|^{2} \mathrm{~d}^{n} x} \\
& \leq \frac{\int_{\Omega}\left(\left|\nabla u_{1}\right|^{2}+V u_{1}^{2}\right) \mathrm{d}^{n} x}{\int_{\Omega} u_{1}^{2} \mathrm{~d}^{n} x}=\lambda_{1}(\Omega, V) .
\end{aligned}
$$

\subsection{Gaussian space}

Consider the space $\mathbb{R}^{n}(n \geq 2)$ endowed with the measure $\mathrm{d} \mu=\gamma(x) \mathrm{d}^{n} x$, where

$$
\gamma(x)=(2 \pi)^{-n / 2} e^{-\frac{|x|^{2}}{2}},
$$

is the standard Gaussian density. Since $\gamma(x)$ is a Gauss function we will call $\left(\mathbb{R}^{n}, \mathrm{~d} \mu\right)$ the Gaussian space. For any Lebesgue-measurable $\Omega \subset \mathbb{R}^{n}$ we define the Gaussian perimeter of $\Omega$ by

$$
P_{\mu}(\Omega)=\sup \left\{\int_{\Omega}((\nabla-x) \cdot v(x)) \gamma(x) \mathrm{d} x: v \in C_{0}^{1}\left(\Omega, \mathbb{R}^{n}\right),\|v\|_{\infty} \leq 1\right\} .
$$

If $\partial \Omega$ is sufficiently well-behaved then

$$
P_{\mu}(\Omega)=\int_{\partial \Omega} \gamma(x) \mathrm{d} H^{n-1}
$$

where $H^{n-1}$ is the $(n-1)$-dimensional Hausdorff measure [51]. It has been shown by Borell that in Gaussian space there is an analog to the classical isoperimetric inequality. Yet the sets that minimize the surface (i.e., the Gaussian perimeter) for a given volume (i.e., Gaussian measure) are not balls, as in Euclidean space, but half-spaces [33]. More precisely:

Theorem 4.3 Let $\Omega \subset \mathbb{R}^{n}$ be open and measurable. Let further $\Omega^{\sharp}$ be the half-space $\left\{\vec{x} \in \mathbb{R}^{n}: x_{1}>a\right\}$, where $a \in \mathbb{R}$ is chosen such that $\mu(\Omega)=\mu\left(\Omega^{\sharp}\right)$. Then

$$
P_{\mu}(\Omega) \geq P_{\mu}\left(\Omega^{\sharp}\right)
$$

with equality only if $\Omega=\Omega^{\sharp}$ up to a rotation.

Next we define the Laplace operator for domains in Gaussian space. We choose an open domain $\Omega \subset \mathbb{R}^{n}$ with $\mu(\Omega)<\mu\left(\mathbb{R}^{n}\right)=1$ and consider the function space

$$
H^{1}(\Omega, \mathrm{d} \mu)=\left\{u \in W_{\text {loc }}^{1,1}(\Omega) \text { such that }(u,|\nabla u|) \in L^{2}(\Omega, \mathrm{d} \mu) \times L^{2}(\Omega, \mathrm{d} \mu)\right\}
$$


endowed with the norm

$$
\|u\|_{H^{1}(\Omega, \mathrm{d} \mu)}=\|u\|_{L^{2}(\Omega, \mathrm{d} \mu)}+\|\nabla u\|_{L^{2}(\Omega, \mathrm{d} \mu)} .
$$

We define the quadratic form

$$
h[u]=\int_{\Omega}|\nabla u|^{2} \mathrm{~d} \mu
$$

on the closure of $C_{0}^{\infty}(\Omega)$ in $H^{1}(\Omega, \mathrm{d} \mu)$. Since $H^{1}$ is complete, Dom $h$ is also complete under its form norm, which is equal to $\|\cdot\|_{H^{1}(\Omega, \mathrm{d} \mu)}$. The quadratic form $h$ is therefore closed and associated with a unique positive self-adjoint operator $-\Delta_{G}$. Dom $h$ is embedded compactly in $L^{2}(\Omega, \mathrm{d} \mu)$ and therefore the spectrum of $-\Delta_{G}$ is discrete. Its eigenfunctions and eigenvalues are solutions of the boundary value problem

$$
\begin{aligned}
-\sum_{j=1}^{n} \frac{\partial}{\partial x_{j}}\left(\gamma(x) \frac{\partial}{\partial x_{j}} u\right) & =\lambda \gamma(x) u & & \text { in } \Omega, \\
u & =0 & & \text { on } \partial \Omega .
\end{aligned}
$$

The analog of the Rayleigh-Faber-Krahn inequality for Gaussian Spaces is the following theorem.

Theorem 4.4 Let $\lambda_{1}(\Omega)$ be the lowest eigenvalue of $-\Delta_{G}$ on $\Omega$ and let $\Omega^{\prime}$ be a half-space of the same Gaussian measure as $\Omega$. Then

$$
\lambda_{1}\left(\Omega^{\prime}\right) \leq \lambda_{1}(\Omega)
$$

Equality holds if and only if $\Omega$ itself is a half-space.

\subsection{Spaces of constant curvature}

Differential operators can not only be defined for functions in Euclidean space, but also for the more general case of functions on Riemannian manifolds. It is therefore natural to ask whether the isoperimetric inequalities for the eigenvalues of the Laplacian can be generalized to such settings as well. In this section we will state Rayleigh-FaberKrahn type theorems for the spaces of constant non-zero curvature, i.e., for the sphere and the hyperbolic space. Isoperimetric inequalities for the second Laplace eigenvalue in these curved spaces will be discussed in Section 6.7.

To start with, we define the Laplacian in hyperbolic space as a self-adjoint operator by means of the quadratic form approach. We realize $\mathbb{H}^{n}$ as the open unit ball $B=\left\{\left(x_{1}, \ldots, x_{n}\right): \sum_{j=1}^{n} x_{j}^{2}<1\right\}$ endowed with the metric

$$
d s^{2}=\frac{4|d x|^{2}}{\left(1-|x|^{2}\right)^{2}}
$$


and the volume element

$$
d V=\frac{2^{n} \mathrm{~d}^{n} x}{\left(1-|x|^{2}\right)^{n}}
$$

where $|\cdot|$ denotes the Euclidean norm. Let $\Omega \subset \mathbb{H}^{n}$ be an open domain and assume that it is bounded in the sense that $\Omega$ does not touch the boundary of $B$. The quadratic form of the Laplace operator in hyperbolic space is the closure of

$$
h[u]=\int_{\Omega} g^{i j}\left(\partial_{i} u\right)\left(\partial_{j} u\right) \mathrm{d} V, \quad u \in C_{0}^{\infty}(\Omega) .
$$

It is easy to see that the form (4.6) is indeed closeable: Since $\Omega$ does not touch the boundary of $B$, the metric coefficients $g^{i j}$ are bounded from above on $\Omega$. They are also bounded from below by $g^{i j} \geq 4$. Consequently, the form norms of $h$ and its Euclidean counterpart, which is the right hand side of (4.6) with $g^{i j}$ replaced by $\delta^{i j}$, are equivalent. Since the 'Euclidean' form is well known to be closeable, $h$ must also be closeable.

By standard spectral theory, the closure of $h$ induces an unique positive self-adjoint operator $-\Delta_{\mathbb{H}}$ which we call the Laplace operator in hyperbolic space. Equivalence between corresponding norms in Euclidean and hyperbolic space implies that the imbedding Dom $h \rightarrow L^{2}(\Omega, \mathrm{d} V)$ is compact and thus the spectrum of $-\Delta_{\mathbb{H}}$ is discrete. For its lowest eigenvalue the following Rayleigh-Faber-Krahn inequality holds.

Theorem 4.5 Let $\Omega \subset \mathbb{H}^{n}$ be an open bounded domain with smooth boundary and $\Omega^{\star} \subset \mathbb{H}^{n}$ an open geodesic ball of the same measure. Denote by $\lambda_{1}(\Omega)$ and $\lambda_{1}\left(\Omega^{\star}\right)$ the lowest eigenvalue of the Dirichlet-Laplace operator on the respective domain. Then

$$
\lambda_{1}\left(\Omega^{\star}\right) \leq \lambda_{1}(\Omega)
$$

with equality only if $\Omega$ itself is a geodesic ball.

The Laplace operator $-\Delta_{\mathbb{S}}$ on a domain which is contained in the unit sphere $\mathbb{S}^{n}$ can be defined in a completely analogous fashion to $-\Delta_{\mathbb{H}}$ by just replacing the metric $g^{i j}$ in (4.6) by the metric of $\mathbb{S}^{n}$.

Theorem 4.6 Let $\Omega \subset \mathbb{S}^{n}$ be an open bounded domain with smooth boundary and $\Omega^{\star} \subset \mathbb{S}^{n}$ an open geodesic ball of the same measure. Denote by $\lambda_{1}(\Omega)$ and $\lambda_{1}\left(\Omega^{\star}\right)$ the lowest eigenvalue of the Dirichlet-Laplace operator on the respective domain. Then

$$
\lambda_{1}\left(\Omega^{\star}\right) \leq \lambda_{1}(\Omega)
$$

with equality only if $\Omega$ itself is a geodesic ball.

The proofs of the above theorems are similar to the proof for the Euclidean case and will be omitted here. A more general Rayleigh-Faber-Krahn theorem for the Laplace operator on Riemannian manifolds and its proof can be found in the book of Chavel [42]. 
4.5 Robin boundary conditions

Yet another generalization of the Rayleigh-Faber-Krahn inequality holds for the boundary value problem

$$
\begin{aligned}
-\sum_{j=1}^{n} \frac{\partial^{2}}{\partial x_{j}^{2}} u=\lambda u & \text { in } \Omega, \\
\frac{\partial u}{\partial v}+\beta u=0 & \text { on } \partial \Omega,
\end{aligned}
$$

on a bounded Lipschitz domain $\Omega \subset \mathbb{R}^{n}$ with the outer unit normal $v$ and some constant $\beta>0$. This so-called Robin boundary value problem can be interpreted as a mathematical model for a vibrating membrane whose edge is coupled elastically to some fixed frame. The parameter $\beta$ indicates how tight this binding is and the eigenvalues of (4.7) correspond the the resonant vibration frequencies of the membrane. They form a sequence $0<\lambda_{1}<\lambda_{2} \leq \lambda_{3} \leq \ldots$ (see, e.g., [75]).

The Robin problem (4.7) is more complicated than the corresponding Dirichlet problem for several reasons. For example, the very useful property of domain monotonicity does not hold for the eigenvalues of the Robin-Laplacian. That is, if one enlarges the domain $\Omega$ in a certain way, the eigenvalues may go up. It is known though, that a very weak form of domain monotonicity holds, namely that $\lambda_{1}(B) \leq \lambda_{1}(\Omega)$ if $B$ is ball that contains $\Omega$. Another difficulty of the Robin problem, compared to the Dirichlet case, is that the level sets of the eigenfunctions may touch the boundary. This makes it impossible, for example, to generalize the proof of the Rayleigh-Faber-Krahn inequality in a straightforward way. Nevertheless, such an isoperimetric inequality holds, as proven by Daners:

Theorem 4.7 Let $\Omega \subset \mathbb{R}^{n}(n \geq 2)$ be a bounded Lipschitz domain, $\beta>0$ a constant and $\lambda_{1}(\Omega)$ the lowest eigenvalue of (4.7). Then $\lambda_{1}\left(\Omega^{\star}\right) \leq \lambda_{1}(\Omega)$.

For the proof of Theorem 4.7, which is not short, we refer the reader to [44].

\subsection{Bibliographical remarks}

i) The Rayleigh-Faber-Krahn inequality is an isoperimetric inequality concerning the lowest eigenvalue of the Laplacian, with Dirichlet boundary condition, on a bounded domain in $\mathbb{R}^{n}(n \geq 2)$. Let $0<\lambda_{1}(\Omega)<\lambda_{2}(\Omega) \leq \lambda_{3}(\Omega) \leq \ldots$ be the Dirichlet eigenvalues of the Laplacian in $\Omega \subset \mathbb{R}^{n}$, i.e.,

$$
\begin{aligned}
& -\Delta u=\lambda u \text { in } \Omega, \\
& u=0 \text { on the boundary of } \Omega .
\end{aligned}
$$

If $n=2$, the Dirichlet eigenvalues are proportional to the square of the eigenfrequencies of an elastic, homogeneous, vibrating membrane with fixed boundary. The Rayleigh-Faber-Krahn inequality for the membrane (i.e., $n=2)$ states that

$$
\lambda_{1} \geq \frac{\pi j_{0,1}^{2}}{A},
$$


where $j_{0,1}=2.4048 \ldots$ is the first zero of the Bessel function of order zero, and $A$ is the area of the membrane. Equality is obtained if and only if the membrane is circular. In other words, among all membranes of given area, the circle has the lowest fundamental frequency. This inequality was conjectured by Lord Rayleigh (see [88], pp. 339-340). In 1918, Courant (see R. Courant, Math. Z. 1, 321-328 (1918)) proved the weaker result that among all membranes of the same perimeter $L$ the circular one yields the least lowest eigenvalue, i.e.,

$$
\lambda_{1} \geq \frac{4 \pi^{2} j_{0,1}^{2}}{L^{2}},
$$

with equality if and only if the membrane is circular. Rayleigh's conjecture was proven independently by Faber [50] and Krahn [64]. The corresponding isoperimetric inequality in dimension $n$,

$$
\lambda_{1}(\Omega) \geq\left(\frac{1}{|\Omega|}\right)^{2 / n} C_{n}^{2 / n} j_{n / 2-1,1},
$$

was proven by Krahn [65]. Here $j_{m, 1}$ is the first positive zero of the Bessel function $J_{m},|\Omega|$ is the volume of the domain, and $C_{n}=\pi^{n / 2} / \Gamma(n / 2+1)$ is the volume of the $n$-dimensional unit ball. Equality is attained if and only if $\Omega$ is a ball. For more details see, R.D. Benguria, Rayleigh-Faber-Krahn Inequality, in Encyclopaedia of Mathematics, Supplement III, Managing Editor: M. Hazewinkel, Kluwer Academic Publishers, pp. 325-327, (2001).

ii) A natural question to ask concerning the Rayleigh-Faber-Krahn inequality is the question of stability. If the lowest eigenvalue of a domain $\Omega$ is within $\epsilon$ (positive and sufficiently small) of the isoperimetric value $\lambda_{1}\left(\Omega^{*}\right)$, how close is the domain $\Omega$ to being a ball? The problem of stability for (convex domains) concerning the Rayleigh-Faber-Krahn inequality was solved by Antonios Melas (see, A. D. Melas, The stability of some eigenvalue estimates, J. Differential Geom. 36, 19-33 (1992)). In the same reference, Melas also solved the analogous stability problem for convex domains with respect to the PPW inequality (see Chapter 6 below). The work of Melas has been extended to the case of the Szegö-Weinberger inequality (for the first nontrivial Neumann eigenvalue) by Y.-Y. Xu, The first nonzero eigenvalue of Neumann problem on Riemannian manifolds, J. Geom. Anal. 5, 151-165 (1995), and to the case of the PPW inequality on spaces of constant curvature by A. Avila, Stability results for the first eigenvalue of the Laplacian on domains in space forms, J. Math. Anal. Appl. 267, 760-774 (2002). In this connection it is worth mentioning related results on the isoperimetric inequality of R. Hall, A quantitative isoperimetric inequality in n-dimensional space, J. Reine Angew Math. 428, 161-176 (1992), as well as recent results of Maggi, Pratelli and Fusco (recently reviewed by F. Maggi in Bull. Amer. Math. Soc. 45, 367-408 (2008)).

iii) The analog of the Faber-Krahn inequality for domains in the sphere $\mathbb{S}^{n}$ was proven by Sperner, Emanuel, Jr. Zur Symmetrisierung von Funktionen auf Sphären, Math. Z. 134, 317-327 (1973).

iv) For isoperimetric inequalities for the lowest eigenvalue of the Laplace-Beltrami operator on manifolds, see, e.g., the book by Chavel, Isaac, Eigenvalues in 
Riemannian geometry. Pure and Applied Mathematics, 115. Academic Press, Inc., Orlando, FL, 1984, (in particular Chapters IV and V), and also the articles, Chavel, I. and Feldman, E. A. Isoperimetric inequalities on curved surfaces. Adv. in Math. 37, 83-98 (1980), and Bandle, Catherine, Konstruktion isoperimetrischer Ungleichungen der mathematischen Physik aus solchen der Geometrie, Comment. Math. Helv. 46, 182-213 (1971).

\section{The Szegö-Weinberger inequality}

In analogy to the Rayleigh-Faber-Krahn inequality for the Dirichlet-Laplacian one may ask which shape of a domain maximizes certain eigenvalues of the Laplace operator with Neumann boundary conditions. Of course, this question is trivial for the lowest Neumann eigenvalue, which is always zero. In 1952 Kornhauser and Stakgold [63] conjectured that the ball maximizes the first non-zero Neumann eigenvalue among all domains of the same volume. This was first proven in 1954 by Szegö [97] for twodimensional simply connected domains, using conformal mappings. Two years later his result was generalized general domains in any dimension by Weinberger [103], who came up with a new strategy for the proof.

Although the Szegö-Weinberger inequality appears to be the analog for Neumann eigenvalues of the Rayleigh-Faber-Krahn inequality, its proof is completely different. The reason is that the first non-trivial Neumann eigenfunction must be orthogonal to the constant function, and thus it must have a change of sign. The simple symmetrization procedure that is used to establish the Rayleigh-Faber-Krahn inequality can therefore not work.

In general, when dealing with Neumann problems, one has to take into account that the spectrum of the respective Laplace operator on a bounded domain is very unstable under perturbations. One can change the spectrum arbitrarily much by only a slight modification of the domain, and if the boundary is not smooth enough, the Laplacian may even have essential spectrum. A sufficient condition for the spectrum of $-\Delta_{N}^{\Omega}$ to be purely discrete is that $\Omega$ is bounded and has a Lipschitz boundary [46]. We write $0=\mu_{0}(\Omega)<\mu_{1}(\Omega) \leq \mu_{2}(\Omega) \leq \ldots$ for the sequence of Neumann eigenvalues on such a domain $\Omega$.

Theorem 5.1 (Szegö-Weinberger inequality) Let $\Omega \subset \mathbb{R}^{n}$ be an open bounded domain with smooth boundary such that the Laplace operator on $\Omega$ with Neumann boundary conditions has purely discrete spectrum. Then

$$
\mu_{1}(\Omega) \leq \mu_{1}\left(\Omega^{\star}\right)
$$

where $\Omega^{\star} \subset \mathbb{R}^{n}$ is a ball with the same $n$-volume as $\Omega$. Equality holds if and only if $\Omega$ itself is a ball.

Proof By a standard separation of variables one shows that $\mu_{1}\left(\Omega^{\star}\right)$ is $n$-fold degenerate and that a basis of the corresponding eigenspace can be written in the form $\left\{g(r) r_{j} r^{-1}\right\}_{j=1, \ldots, n}$. The function $g$ can be chosen to be positive and satisfies the differential equation 


$$
g^{\prime \prime}+\frac{n-1}{r} g^{\prime}+\left(\mu_{1}\left(\Omega^{\star}\right)-\frac{n-1}{r^{2}}\right) g=0, \quad 0<r<r_{1},
$$

where $r_{1}$ is the radius of $\Omega^{\star}$. Further, $g(r)$ vanishes at $r=0$ and its derivative has its first zero at $r=r_{1}$. We extend $g$ by defining $g(r)=\lim _{r^{\prime} \uparrow r_{1}} g\left(r^{\prime}\right)$ for $r \geq r_{1}$. Then $g$ is differentiable on $\mathbb{R}$ and if we set $f_{j}(\vec{r}):=g(r) r_{j} r^{-1}$ then $f_{j} \in W^{1,2}(\Omega)$ for $j=1 \ldots, n$. To apply the min-max principle with $f_{j}$ as a test function for $\mu_{1}(\Omega)$ we have to make sure that $f_{j}$ is orthogonal to the first (trivial) eigenfunction, i.e., that

$$
\int_{\Omega} f_{j} \mathrm{~d}^{n} r=0, \quad j=1, \ldots, n
$$

We argue that this can be achieved by some shift of the domain $\Omega$ : Since $\Omega$ is bounded we can find a ball $B$ that contains $\Omega$. Now define the vector field $\vec{b}: \mathbb{R}^{n} \rightarrow \mathbb{R}^{n}$ by its components

$$
b_{j}(\vec{v})=\int_{\Omega+\vec{v}} f_{j}(\vec{r}) \mathrm{d}^{n} r, \quad \vec{v} \in \mathbb{R}^{n} .
$$

For $\vec{v} \in \partial B$ we have

$$
\begin{aligned}
\vec{v} \cdot \vec{b}(\vec{v}) & =\int_{\Omega+\vec{v}} \frac{\vec{v} \cdot \vec{r}}{r} g(r) \mathrm{d}^{n} r \\
& =\int_{\Omega} \frac{\vec{v} \cdot(\vec{r}+\vec{v})}{|\vec{r}+\vec{v}|} g(|\vec{r}+\vec{v}|) \mathrm{d}^{n} r \\
& \geq \int_{\Omega} \frac{|\vec{v}|^{2}-|v| \cdot|r|}{|\vec{r}+\vec{v}|} g(|\vec{r}+\vec{v}|) \mathrm{d}^{n} r>0 .
\end{aligned}
$$

Thus $\vec{b}$ is a vector field that points outwards on every point of $\partial B$. By an application of the Brouwer's fixed-point theorem (see Theorem 10.3 in the Appendix) this means that $\vec{b}\left(\vec{v}_{0}\right)=0$ for some $\vec{v}_{0} \in B$. Thus, if we shift $\Omega$ by this vector, condition (5.3) is satisfied and we can apply the min-max principle with the $f_{j}$ as test functions for the first non-zero eigenvalue:

$$
\begin{aligned}
\mu_{1}(\Omega) & \leq \frac{\int_{\Omega}\left|\nabla f_{j}\right| \mathrm{d}^{n} r}{\int_{\Omega} f_{j}^{2} \mathrm{~d}^{n} r} \\
& =\frac{\int_{\Omega}\left(g^{\prime 2}(r) r_{j}^{2} r^{-2}+g^{2}(r)\left(1-r_{j}^{2} r^{-2}\right) r^{-2}\right) \mathrm{d}^{n} r}{\int_{\Omega} g^{2} r_{j}^{2} r^{-2} \mathrm{~d}^{n} r} .
\end{aligned}
$$

We multiply each of these inequalities by the denominator and sum up over $j$ to obtain

$$
\mu_{1}(\Omega) \leq \frac{\int_{\Omega} B(r) \mathrm{d}^{n} r}{\int_{\Omega} g^{2}(r) \mathrm{d}^{n} r}
$$


with $B(r)=g^{\prime 2}(r)+(n-1) g^{2}(r) r^{-2}$. Since $r_{1}$ is the first zero of $g^{\prime}$, the function $g$ is non-decreasing. The derivative of $B$ is

$$
B^{\prime}=2 g^{\prime} g^{\prime \prime}+2(n-1)\left(r g g^{\prime}-g^{2}\right) r^{-3} \text {. }
$$

For $r \geq r_{1}$ this is clearly negative since $g$ is constant there. For $r<r_{1}$ we can use equation (5.2) to show that

$$
B^{\prime}=-2 \mu_{1}\left(\Omega^{\star}\right) g g^{\prime}-(n-1)\left(r g^{\prime}-g\right)^{2} r^{-3}<0 .
$$

If the following we will use the method of rearrangements, which was described in Chapter 3. To avoid confusions, we use a more precise notation at this point: We introduce $B_{\Omega}: \Omega \rightarrow \mathbb{R}, B_{\Omega}(\vec{r})=B(r)$ and analogously $g_{\Omega}: \Omega \rightarrow \mathbb{R}, g_{\Omega}(\vec{r})=g(r)$. Then equation (5.4) yields, using Theorem 3.7 in the third step:

$$
\mu_{1}(\Omega) \leq \frac{\int_{\Omega} B_{\Omega}(\vec{r}) \mathrm{d}^{n} r}{\int_{\Omega} g_{\Omega}^{2}(\vec{r}) \mathrm{d}^{n} r}=\frac{\int_{\Omega^{\star}} B_{\Omega^{\star}}^{\star}(\vec{r}) \mathrm{d}^{n} r}{\int_{\Omega^{\star}} g_{\star}^{2}(\vec{r}) \mathrm{d}^{n} r} \leq \frac{\int_{\Omega^{\star}} B(r) \mathrm{d}^{n} r}{\int_{\Omega^{\star}} g^{2}(r) \mathrm{d}^{n} r}=\mu_{1}\left(\Omega^{\star}\right)
$$

Equality holds obviously if $\Omega$ is a ball. In any other case the third step in (5.5) is a strict inequality.

It is rather straightforward to generalize the Szegö-Weinberger inequality to domains in hyperbolic space. For domains on spheres, on the other hand, the corresponding inequality has not been established yet in full generality. At present, the most general result is due to Ashbaugh and Benguria: In [12] they show that an ana$\log$ of the Szegö-Weinberger inequality holds for domains that are contained in a hemisphere.

\subsection{Bibliographical remarks}

i) In 1952, Kornhauser and Stakgold [63] conjectured that the lowest nontrivial Neumann eigenvalue for a smooth bounded domain $\Omega$ in $\mathbb{R}^{2}$ satisfies the isoperimetric inequality

$$
\mu_{1}(\Omega) \leq \mu_{1}\left(\Omega^{*}\right)=\frac{\pi p^{2}}{A}
$$

where $\Omega^{*}$ is a disk with the same area as $\Omega$, and $p=1.8412 \ldots$ is the first positive zero of the derivative of the Bessel function $J_{1}$. This conjecture was proven by $\mathrm{G}$. Szegö in 1954, using conformal maps [97]. The extension to $n$ dimensions was proven by $\mathrm{H}$. Weinberger [103].

ii) For the case of mixed boundary conditions, Marie-Helene Bossel [Membranes élastiquement liées inhomogénes ou sur une surface: une nouvelle extension du théoreme isopérimétrique de Rayleigh-Faber-Krahn, Z. Angew. Math. Phys. 39, 733-742 (1988)] proved the analog of the Rayleigh-Faber-Krahn inequality. 
iii) Very recently, A. Girouard, N. Nadirashvili and I. Polterovich proved that the second positive eigenvalue of a bounded simply connected planar domain of a given area does not exceed the first positive Neumann eigenvalue on a disk of a twice smaller area (see, Maximization of the second positive Neumann eigenvalue for planar domains, J. Differential Geom. 83, 637-662 (2009)). For a review of optimization of eigenvalues with respect to the geometry of the domain, see the recent monograph of A. Henrot [60].

iv) In the Bibliographical Remarks of Section 4 (see Section 4.6, ii)) we discussed the stability results of A. Melas for the Rayleigh-Faber-Krahn inequality. In the same vein, recently L. Brasco and A. Pratellli, Sharp Stability of some Spectral Inequalities, preprint (2011), have proven related stability results for the Szegö-Weinberger inequality. Moreover, these authors have also proven stability results for the E. KrahnP. Szego inequality, which says that among all sets of a given measure (in Euclidean Space) the disjoint union of two balls with the same radius minimizes the second eigenvalue of the Dirichlet Laplacian.

\section{The Payne-Pólya-Weinberger inequality}

\subsection{Introduction}

A further isoperimetric inequality is concerned with the second eigenvalue of the Dirichlet-Laplacian on bounded domains. In 1955 Payne, Pólya and Weinberger (PPW) showed that for any open bounded domain $\Omega \subset \mathbb{R}^{2}$ the bound $\lambda_{2}(\Omega) / \lambda_{1}(\Omega) \leq$ 3 holds $[83,84]$. Based on exact calculations for simple domains they also conjectured that the ratio $\lambda_{2}(\Omega) / \lambda_{1}(\Omega)$ is maximized when $\Omega$ is a circular disk, i.e., that

$$
\frac{\lambda_{2}(\Omega)}{\lambda_{1}(\Omega)} \leq \frac{\lambda_{2}\left(\Omega^{\star}\right)}{\lambda_{1}\left(\Omega^{\star}\right)}=\frac{j_{1,1}^{2}}{j_{0,1}^{2}} \approx 2.539 \text { for } \Omega \subset \mathbb{R}^{2} .
$$

Here, $j_{n, m}$ denotes the $m^{\text {th }}$ positive zero of the Bessel function $J_{n}(x)$. This conjecture and the corresponding inequalities in $n$ dimensions were proven in 1991 by Ashbaugh and Benguria [9-11]. Since the Dirichlet eigenvalues on a ball are inversely proportional to the square of the ball's radius, the ratio $\lambda_{2}\left(\Omega^{\star}\right) / \lambda_{1}\left(\Omega^{\star}\right)$ does not depend on the size of $\Omega^{\star}$. Thus we can state the PPW inequality in the following form:

Theorem 6.1 (Payne-Pólya-Weinberger inequality) Let $\Omega \subset \mathbb{R}^{n}$ be an open bounded domain and $S_{1} \subset \mathbb{R}^{n}$ a ball such that $\lambda_{1}(\Omega)=\lambda_{1}\left(S_{1}\right)$. Then

$$
\lambda_{2}(\Omega) \leq \lambda_{2}\left(S_{1}\right)
$$

with equality if and only if $\Omega$ is a ball.

Here the subscript 1 on $S_{1}$ reflects the fact that the ball $S_{1}$ has the same first Dirichlet eigenvalue as the original domain $\Omega$. The inequalities (6.1) and (6.2) are equivalent in Euclidean space in view of the mentioned scaling properties of the eigenvalues. 
Yet when one considers possible extensions of the PPW inequality to other settings, where $\lambda_{2} / \lambda_{1}$ varies with the radius of the ball, it turns out that an estimate in the form of Theorem 6.1 is the more natural result. In the case of a domain on a hemisphere, for example, $\lambda_{2} / \lambda_{1}$ on balls is an increasing function of the radius. But by the RayleighFaber-Krahn inequality for spheres the radius of $S_{1}$ is smaller than the one of the spherical rearrangement $\Omega^{\star}$. This means that an estimate in the form of Theorem 6.1, interpreted as

$$
\frac{\lambda_{2}(\Omega)}{\lambda_{1}(\Omega)} \leq \frac{\lambda_{2}\left(S_{1}\right)}{\lambda_{1}\left(S_{1}\right)}, \quad \Omega, S_{1} \subset \mathbb{S}^{n},
$$

is stronger than an inequality of the type (6.1).

On the other hand, we will see that in the hyperbolic space $\lambda_{2} / \lambda_{1}$ on balls is a strictly decreasing function of the radius. In this case we can apply the following argument to see that an estimate of the type (6.1) cannot possibly hold true: Consider a domain $\Omega$ that is constructed by attaching very long and thin tentacles to the ball $B$. Then the first and second eigenvalues of the Laplacian on $\Omega$ are arbitrarily close to the ones on $B$. The spherical rearrangement of $\Omega$ though can be considerably larger than $B$. This means that

$$
\frac{\lambda_{2}(\Omega)}{\lambda_{1}(\Omega)} \approx \frac{\lambda_{2}(B)}{\lambda_{1}(B)}>\frac{\lambda_{2}\left(\Omega^{\star}\right)}{\lambda_{1}\left(\Omega^{\star}\right)}, \quad B, \Omega \subset \mathbb{H}^{n},
$$

clearly ruling out any inequality in the form of (6.1).

The proof of the PPW inequality (6.2) is somewhat similar to that of the SzegöWeinberger inequality (see Chapter 5), but considerably more difficult. The additional complications mainly stem from the fact that in the Dirichlet case the first eigenfunction of the Laplacian is not known explicitly, while in the Neumann case it is just constant. We will give the full proof of the PPW inequality in the following three sections. Since it is quite long, a brief outline is in order:

The proof is organized in six steps. In the first one we use the min-max principle to derive an estimate for the eigenvalue gap $\lambda_{2}(\Omega)-\lambda_{1}(\Omega)$, depending on a test function for the second eigenvalue. In the second step we define such a function and then show in the third step that it actually satisfies all requirements to be used in the gap formula. In the fourth step we put the test function into the gap inequality and then estimate the result with the help of rearrangement techniques. These depend on the monotonicity properties of two functions $g$ and $B$, which are to be defined in the proof, and on a Chiti comparison argument. The later is a special comparison result which establishes a crossing property between the symmetric decreasing rearrangement of the first eigenfunction on $\Omega$ and the first eigenfunction on $S_{1}$. We end up with the inequality $\lambda_{2}(\Omega)-\lambda_{1}(\Omega) \leq \lambda_{2}\left(S_{1}\right)-\lambda_{1}\left(S_{1}\right)$, which yields (6.2). In the remaining two steps we prove the mentioned monotonicity properties and the Chiti comparison result. We remark that from the Rayleigh-Faber-Krahn inequality follows $S_{1} \subset \Omega^{\star}$, a fact that is used in the proof of the Chiti comparison result. Although it enters in a rather subtle manner, the Rayleigh-Faber-Krahn inequality is an important ingredient of the proof of the PPW inequality. 


\subsection{Proof of the Payne-Pólya-Weinberger inequality}

First step: We derive the 'gap formula' for the first two eigenvalues of the DirichletLaplacian on $\Omega$. We call $u_{1}: \Omega \rightarrow \mathbb{R}^{+}$the positive normalized first eigenfunction of $-\Delta_{\Omega}^{D}$. To estimate the second eigenvalue we will use the test function $P u_{1}$, where $P: \Omega \rightarrow \mathbb{R}$ is is chosen such that $P u_{1}$ is in the form domain of $-\Delta_{\Omega}^{D}$ and

$$
\int_{\Omega} P u_{1}^{2} \mathrm{~d} r^{n}=0
$$

Then we conclude from the min-max principle that

$$
\begin{aligned}
\lambda_{2}(\Omega)-\lambda_{1}(\Omega) & \leq \frac{\int_{\Omega}\left(\left|\nabla\left(P u_{1}\right)\right|^{2}-\lambda_{1} P^{2} u_{1}^{2}\right) \mathrm{d} r^{n}}{\int_{\Omega} P^{2} u_{1}^{2} \mathrm{~d} r^{n}} \\
& =\frac{\int_{\Omega}\left(|\nabla P|^{2} u_{1}^{2}+\left(\nabla P^{2}\right) u_{1} \nabla u_{1}+P^{2}\left|\nabla u_{1}\right|^{2}-\lambda_{1} P^{2} u_{1}^{2}\right) \mathrm{d} r^{n}}{\int_{\Omega} P^{2} u_{1}^{2} \mathrm{~d} r^{n}}
\end{aligned}
$$

If we perform an integration by parts on the second summand in the numerator of (6.4), we see that all summands except the first cancel. We obtain the gap inequality

$$
\lambda_{2}(\Omega)-\lambda_{1}(\Omega) \leq \frac{\int_{\Omega}|\nabla P|^{2} u_{1}^{2} \mathrm{~d} r^{n}}{\int_{\Omega} P^{2} u_{1}^{2} \mathrm{~d} r^{n}}
$$

Second step: We need to fix the test function $P$. Our choice will be dictated by the requirement that equality should hold in (6.5) if $\Omega$ is a ball, i.e., if $\Omega=S_{1}$ up to translations. We assume that $S_{1}$ is centered at the origin of our coordinate system and call $R_{1}$ its radius. We write $z_{1}(r)$ for the first eigenfunction of the Dirichlet Laplacian on $S_{1}$. This function is spherically symmetric with respect to the origin and we can take it to be positive and normalized in $L^{2}\left(S_{1}\right)$. The second eigenvalue of $-\Delta_{S_{1}}^{D}$ in $n$ dimensions is $n$-fold degenerate and a basis of the corresponding eigenspace can be written in the form $z_{2}(r) r_{j} r^{-1}$ with $z_{2} \geq 0$ and $j=1, \ldots, n$. This is the motivation to choose not only one test function $P$, but rather $n$ functions $P_{j}$ with $j=1, \ldots, n$. We set

$$
P_{j}=r_{j} r^{-1} g(r)
$$

with

$$
g(r)= \begin{cases}\frac{z_{2}(r)}{z_{1}(r)} & \text { for } r<R_{1}, \\ \lim _{r^{\prime} \uparrow R_{1}} \frac{z_{2}\left(r^{\prime}\right)}{z_{1}\left(r^{\prime}\right)} & \text { for } r \geq R_{1} .\end{cases}
$$

We note that $P_{j} u_{1}$ is a second eigenfunction of $-\Delta_{\Omega}^{D}$ if $\Omega$ is a ball which is centered at the origin.

Third step: It is necessary to verify that the $P_{j} u_{1}$ are admissible test functions. First, we have to make sure that condition (6.3) is satisfied. We note that $P_{j}$ changes when 
$\Omega$ (and $u_{1}$ with it) is shifted in $\mathbb{R}^{n}$. Since these shifts do not change $\lambda_{1}(\Omega)$ and $\lambda_{2}(\Omega)$, it is sufficient to show that $\Omega$ can be moved in $\mathbb{R}^{n}$ such that (6.3) is satisfied for all $j \in\{1, \ldots, n\}$. To this end we define the function

$$
\vec{b}(\vec{v})=\int_{\Omega+\vec{v}} u_{1}^{2}(|\vec{r}-\vec{v}|) \frac{\vec{r}}{r} g(r) \mathrm{d} r^{n} \quad \text { for } \vec{v} \in \mathbb{R}^{n} .
$$

Since $\Omega$ is a bounded domain, we can choose some closed ball $D$, centered at the origin, such that $\Omega \subset D$. Then for every $\vec{v} \in \partial D$ we have

$$
\begin{aligned}
\vec{v} \cdot \vec{b}(\vec{v}) & =\int_{\Omega} \vec{v} \cdot u_{1}^{2}(r) \frac{\vec{r}+\vec{v}}{|\vec{r}+\vec{v}|} g(|\vec{r}+\vec{v}|) \mathrm{d} r^{n} \\
& >\int_{\Omega} u_{1}^{2}(r) \frac{|\vec{v}|^{2}-|\vec{v}| \cdot|\vec{r}|}{|\vec{r}+\vec{v}|} g(|\vec{r}+\vec{v}|) \mathrm{d} r^{n}>0
\end{aligned}
$$

Thus the continuous vector-valued function $\vec{b}(\vec{v})$ points strictly outwards everywhere on $\partial D$. By Theorem 10.3, which is a consequence of the Brouwer fixed-point theorem, there is some $\vec{v}_{0} \in D$ such that $\vec{b}\left(\vec{v}_{0}\right)=0$. Now we shift $\Omega$ by this vector, i.e., we replace $\Omega$ by $\Omega-\vec{v}_{0}$ and $u_{1}$ by the first eigenfunction of the shifted domain. Then the test functions $P_{j} u_{1}$ satisfy the condition (6.3).

The second requirement on $P_{j} u_{1}$ is that it must be in the form domain of $-\Delta_{\Omega}^{D}$, i.e., in $H_{0}^{1}(\Omega)$ : Since $u_{1} \in H_{0}^{1}(\Omega)$ there is a sequence $\left\{v_{n} \in C^{1}(\Omega)\right\}_{n \in \mathbb{N}}$ of functions with compact support such that $|\cdot|_{h}-\lim _{n \rightarrow \infty} v_{n}=u_{1}$, using the definition (4.1) of $|\cdot|_{h}$. The functions $P_{j} v_{n}$ also have compact support and one can check that $P_{j} v_{n} \in C^{1}(\Omega)\left(P_{j}\right.$ is continuously differentiable since $\left.g^{\prime}\left(R_{1}\right)=0\right)$. We have $|\cdot|_{h}-\lim _{n \rightarrow \infty} P_{j} v_{n}=P_{j} u_{1}$ and thus $P_{j} u_{1} \in H_{0}^{1}(\Omega)$.

Fourth step: We multiply the gap inequality (6.5) by $\int P^{2} u_{1}^{2} \mathrm{~d} x$ and put in our special choice of $P_{j}$ to obtain

$$
\begin{aligned}
\left(\lambda_{2}-\lambda_{1}\right) \int_{\Omega} \frac{r_{j}^{2}}{r^{2}} g^{2}(r) u_{1}^{2}(r) \mathrm{d} r^{n} & \leq \int_{\Omega}\left|\nabla\left(\frac{r_{j}}{r} g(r)\right)\right|^{2} u_{1}^{2}(r) \mathrm{d} r^{n} \\
& =\int_{\Omega}\left(\left|\nabla \frac{r_{j}}{r}\right|^{2} g^{2}(r)+\frac{r_{j}^{2}}{r^{2}} g^{\prime}(r)^{2}\right) u_{1}^{2}(r) \mathrm{d} r^{n} .
\end{aligned}
$$

Now we sum these inequalities up over $j=1, \ldots, n$ and then divide again by the integral on the left hand side to get

$$
\lambda_{2}(\Omega)-\lambda_{1}(\Omega) \leq \frac{\int_{\Omega} B(r) u_{1}^{2}(r) \mathrm{d} r^{n}}{\int_{\Omega} g^{2}(r) u_{1}^{2}(r) \mathrm{d} r^{n}}
$$

with

$$
B(r)=g^{\prime}(r)^{2}+(n-1) r^{-2} g(r)^{2} .
$$


If the following we will use the method of rearrangements, which was described in Chapter 3. To avoid confusions, we use a more precise notation at this point: We introduce $B_{\Omega}: \Omega \rightarrow \mathbb{R}, B_{\Omega}(\vec{r})=B(r)$ and analogously $g_{\Omega}: \Omega \rightarrow \mathbb{R}, g_{\Omega}(\vec{r})=g(r)$. Then equation (6.6) can be written as

$$
\lambda_{2}(\Omega)-\lambda_{1}(\Omega) \leq \frac{\int_{\Omega} B_{\Omega}(\vec{r}) u_{1}^{2}(\vec{r}) \mathrm{d} r^{n}}{\int_{\Omega} g_{\Omega}^{2}(\vec{r}) u_{1}^{2}(\vec{r}) \mathrm{d} r^{n}} .
$$

Then by Theorem 3.8 the following inequality is also true:

$$
\lambda_{2}(\Omega)-\lambda_{1}(\Omega) \leq \frac{\int_{\Omega^{\star}} B_{\Omega^{\star}}(\vec{r}) u_{1}^{\star}(\vec{r})^{2} \mathrm{~d} r^{n}}{\int_{\Omega^{\star}} g_{\Omega_{\star}}^{2}(\vec{r}) u_{1}^{\star}(\vec{r})^{2} \mathrm{~d} r^{n}} .
$$

Next we use the very important fact that $g(r)$ is an increasing function and $B(r)$ is a decreasing function, which we will prove in step five below. These monotonicity properties imply by Theorem 3.7 that $B_{\Omega}^{\star}(\vec{r}) \leq B(r)$ and $g_{\Omega \star}(\vec{r}) \geq g(r)$. Therefore

$$
\lambda_{2}(\Omega)-\lambda_{1}(\Omega) \leq \frac{\int_{\Omega^{\star}} B(r) u_{1}^{\star}(r)^{2} \mathrm{~d} r^{n}}{\int_{\Omega^{\star}} g^{2}(r) u_{1}^{\star}(r)^{2} \mathrm{~d} r^{n}} .
$$

Finally we use the following version of Chiti's comparison theorem to estimate the right hand side of (6.10):

Lemma 6.2 (Chiti comparison result) There is some $r_{0} \in\left(0, R_{1}\right)$ such that

$$
\begin{aligned}
& z_{1}(r) \geq u_{1}^{\star}(r) \quad \text { for } r \in\left(0, r_{0}\right) \text { and } \\
& z_{1}(r) \leq u_{1}^{\star}(r) \quad \text { for } r \in\left(r_{0}, R_{1}\right) .
\end{aligned}
$$

We remind the reader that the function $z_{1}$ denotes the first Dirichlet eigenfunction for the Laplacian defined on $S_{1}$. Applying Lemma 6.2, which will be proven below in step six, to (6.10) yields

$$
\lambda_{2}(\Omega)-\lambda_{1}(\Omega) \leq \frac{\int_{\Omega^{\star}} B(r) z_{1}(r)^{2} \mathrm{~d} r^{n}}{\int_{\Omega^{\star}} g^{2}(r) z_{1}(r)^{2} \mathrm{~d} r^{n}}=\lambda_{2}\left(S_{1}\right)-\lambda_{1}\left(S_{1}\right) .
$$

Since $S_{1}$ was chosen such that $\lambda_{1}(\Omega)=\lambda_{1}\left(S_{1}\right)$ the above relation proves that $\lambda_{2}(\Omega) \leq$ $\lambda_{2}\left(S_{1}\right)$. It remains the question: When does equality hold in (6.2)? It is obvious that equality does hold if $\Omega$ is a ball, since then $\Omega=S_{1}$ up to translations. On the other hand, if $\Omega$ is not a ball, then (for example) the step from (6.10) to (6.11) is not sharp. Thus (6.2) is a strict inequality if $\Omega$ is not a ball.

\subsection{Monotonicity of $B$ and $g$}

Fifth step: We prove that $g(r)$ is an increasing function and $B(r)$ is a decreasing function. In this step we abbreviate $\lambda_{i}=\lambda_{i}\left(S_{1}\right)$. The functions $z_{1}$ and $z_{2}$ are solutions of the differential equations 


$$
\begin{array}{r}
-z_{1}^{\prime \prime}-\frac{n-1}{r} z_{1}^{\prime}-\lambda_{1} z_{1}=0, \\
-z_{2}^{\prime \prime}-\frac{n-1}{r} z_{2}^{\prime}+\left(\frac{n-1}{r^{2}}-\lambda_{2}\right) z_{2}=0
\end{array}
$$

with the boundary conditions

$$
z_{1}^{\prime}(0)=0, \quad z_{1}\left(R_{1}\right)=0, \quad z_{2}(0)=0, \quad z_{2}\left(R_{1}\right)=0 .
$$

We define the function

$$
q(r):= \begin{cases}\frac{r g^{\prime}(r)}{g(r)} & \text { for } r \in\left(0, R_{1}\right), \\ \lim _{r^{\prime} \downarrow 0} q\left(r^{\prime}\right) & \text { for } r=0, \\ \lim _{r^{\prime} \uparrow R_{1}} q\left(r^{\prime}\right) & \text { for } r=R_{1} .\end{cases}
$$

Proving the monotonicity of $B$ and $g$ is thus reduced to showing that $0 \leq q(r) \leq 1$ and $q^{\prime}(r) \leq 0$ for $r \in\left[0, R_{1}\right]$. Using the definition of $g$ and the equations (6.12), one can show that $q(r)$ is a solution of the Riccati differential equation

$$
q^{\prime}=\left(\lambda_{1}-\lambda_{2}\right) r+\frac{(1-q)(q+n-1)}{r}-2 q \frac{z_{1}^{\prime}}{z_{1}} .
$$

It is straightforward to establish the boundary behavior

$$
q(0)=1, \quad q^{\prime}(0)=0, \quad q^{\prime \prime}(0)=\frac{2}{n}\left(\left(1+\frac{2}{n}\right) \lambda_{1}-\lambda_{2}\right)
$$

and

$$
q\left(R_{1}\right)=0 .
$$

Lemma 6.3 For $0 \leq r \leq R_{1}$ we have $q(r) \geq 0$.

Proof Assume the contrary. Then there exist two points $0<s_{1}<s_{2} \leq R_{1}$ such that $q\left(s_{1}\right)=q\left(s_{2}\right)=0$ but $q^{\prime}\left(s_{1}\right) \leq 0$ and $q^{\prime}\left(s_{2}\right) \geq 0$. If $s_{2}<R_{1}$ then the Riccati equation (6.15) yields

$$
0 \geq q^{\prime}\left(s_{1}\right)=\left(\lambda_{1}-\lambda_{2}\right) s_{1}+\frac{n-1}{s_{1}}>\left(\lambda_{1}-\lambda_{2}\right) s_{2}+\frac{n-1}{s_{2}}=q^{\prime}\left(s_{2}\right) \geq 0,
$$

which is a contradiction. If $s_{2}=R_{1}$ then we get a contradiction in a similar way by

$$
0 \geq q^{\prime}\left(s_{1}\right)=\left(\lambda_{1}-\lambda_{2}\right) s_{1}+\frac{n-1}{s_{1}}>\left(\lambda_{1}-\lambda_{2}\right) R_{1}+\frac{n-1}{R_{1}}=3 q^{\prime}\left(R_{1}\right) \geq 0 .
$$

In the following we will analyze the behavior of $q^{\prime}$ according to (6.15), considering $r$ and $q$ as two independent variables. For the sake of a compact notation we will make use of the following abbreviations: 


$$
\begin{aligned}
p(r) & =z_{1}^{\prime}(r) / z_{1}(r) \\
N_{y} & =y^{2}-n+1 \\
Q_{y} & =2 y \lambda_{1}+\left(\lambda_{2}-\lambda_{1}\right) N_{y} y^{-1}-2\left(\lambda_{2}-\lambda_{1}\right) \\
M_{y} & =N_{y}^{2} /(2 y)-(n-2)^{2} y / 2
\end{aligned}
$$

We further define the function

$$
T(r, y):=-2 p(r) y-\frac{(n-2) y+N_{y}}{r}-\left(\lambda_{2}-\lambda_{1}\right) r .
$$

Then we can write $(6.15)$ as

$$
q^{\prime}(r)=T(r, q(r))
$$

The definition of $T(r, y)$ allows us to analyze the Riccati equation for $q^{\prime}$ considering $r$ and $q(r)$ as independent variables. For $r$ going to zero, $p$ is $\mathcal{O}(r)$ and thus

$$
T(r, y)=\frac{1}{r}((n-1+y)(1-y))+\mathcal{O}(r) \text { for } y \text { fixed. }
$$

Consequently,

$$
\begin{array}{ll}
\lim _{r \rightarrow 0} T(r, y)=+\infty & \text { for } 0 \leq y<1 \text { fixed } \\
\lim _{r \rightarrow 0} T(r, y)=0 & \text { for } y=1 \text { and } \\
\lim _{r \rightarrow 0} T(r, y)=-\infty & \text { for } y>1 \text { fixed. }
\end{array}
$$

The partial derivative of $T(r, y)$ with respect to $r$ is given by

$$
T^{\prime}=\frac{\partial}{\partial r} T(r, y)=-2 y p^{\prime}+\frac{(n-2) y}{r^{2}}+\frac{N_{y}}{r^{2}}-\left(\lambda_{2}-\lambda_{1}\right) .
$$

In the points $(r, y)$ where $T(r, y)=0$ we have, by (6.16),

$$
\left.p\right|_{T=0}=-\frac{n-2}{2 r}-\frac{N_{y}}{2 y r}-\frac{\left(\lambda_{2}-\lambda_{1}\right) r}{2 y} .
$$

From (6.12) we get the Riccati equation

$$
p^{\prime}+p^{2}+\frac{n-1}{r} p+\lambda_{1}=0 .
$$

Putting (6.18) into (6.19) and the result into (6.17) yields

$$
\left.T^{\prime}\right|_{T=0}=\frac{M_{y}}{r^{2}}+\frac{\left(\lambda_{2}-\lambda_{1}\right)^{2}}{2 y} r^{2}+Q_{y}
$$

Lemma 6.4 There is some $r_{0}>0$ such that $q(r) \leq 1$ for all $r \in\left(0, r_{0}\right)$ and $q\left(r_{0}\right)<1$. 
Proof Suppose the contrary, i.e., $q(r)$ first increases away from $r=0$. Then, because $q(0)=1$ and $q\left(R_{1}\right)=0$ and because $q$ is continuous and differentiable, we can find two points $s_{1}<s_{2}$ such that $\hat{q}:=q\left(s_{1}\right)=q\left(s_{2}\right)>1$ and $q^{\prime}\left(s_{1}\right)>0>q^{\prime}\left(s_{2}\right)$. Even more, we can chose $s_{1}$ and $s_{2}$ such that $\hat{q}$ is arbitrarily close to one. Writing $\hat{q}=1+\epsilon$ with $\epsilon>0$, we can calculate from the definition of $Q_{y}$ that

$$
Q_{1+\epsilon}=Q_{1}+\epsilon n\left(\lambda_{2}-(1-2 / n) \lambda_{1}\right)+\mathcal{O}\left(\epsilon^{2}\right) .
$$

The term in brackets can be estimated by

$$
\lambda_{2}-(1-2 / n) \lambda_{1}>\lambda_{2}-\lambda_{1}>0 \text {. }
$$

We can also assume that $Q_{1} \geq 0$, because otherwise $q^{\prime \prime}(0)=\frac{2}{n^{2}} Q_{1}<0$ and Lemma 6.4 is immediately true. Thus, choosing $R_{1}$ and $r_{2}$ such that $\epsilon$ is sufficiently small, we can make sure that $Q_{\hat{q}}>0$.

Now consider $T(r, \hat{q})$ as a function of $r$ for our fixed $\hat{q}$. We have $T\left(s_{1}, \hat{q}\right)>0>$ $T\left(s_{2}, \hat{q}\right)$ and the boundary behavior $T(0, \hat{q})=-\infty$. Consequently, $T(r, \hat{q})$ changes its sign at least twice on $\left[0, R_{1}\right]$ and thus we can find two zeros $0<\hat{s}_{1}<\hat{s}_{2}<R_{1}$ of $T(r, \hat{q})$ such that

$$
T^{\prime}\left(\hat{s}_{1}, \hat{q}\right) \geq 0 \text { and } \quad T^{\prime}\left(\hat{s}_{2}, \hat{q}\right) \leq 0 .
$$

But from (6.20), together with $Q_{\hat{q}}>0$, one can see easily that this is impossible, because the right hand side of (6.20) is either positive or increasing (depending on $M_{\hat{q}}$ ). This is a contradiction to our assumption that $q$ first increases away from $r=0$, proving Lemma 6.4 .

Lemma 6.5 For all $0 \leq r \leq R_{1}$ the inequality $q^{\prime}(r) \leq 0$ holds.

Proof Assume the contrary. Then, because of $q(0)=1$ and $q\left(R_{1}\right)=0$, there are three points $s_{1}<s_{2}<s_{3}$ in $\left(0, R_{1}\right)$ with $0<\hat{q}:=q\left(s_{1}\right)=q\left(s_{2}\right)=q\left(s_{3}\right)<1$ and $q^{\prime}\left(s_{1}\right)<0, q^{\prime}\left(s_{2}\right)>0, q^{\prime}\left(s_{3}\right)<0$. Consider the function $T(r, \hat{q})$, which coincides with $q^{\prime}(r)$ at $s_{1}, s_{2}, s_{3}$. Taking into account its boundary behavior at $r=0$, it is clear that $T(r, \hat{q})$ must have at least the sign changes positive-negative-positive-negative. Thus $T(r, \hat{q})$ has at least three zeros $\hat{s}_{1}<\hat{s}_{2}<\hat{s}_{3}$ with the properties

$$
T^{\prime}\left(\hat{s}_{1}, \hat{q}\right) \leq 0, \quad T^{\prime}\left(\hat{s}_{2}, \hat{q}\right) \geq 0, \quad T^{\prime}\left(\hat{s}_{3}, \hat{q}\right) \leq 0
$$

Again one can see from (6.20) that this is impossible, because the term on the right hand side is either a strictly convex or a strictly increasing function of $r$. We conclude that Lemma 6.5 is true.

Altogether we have shown that $0 \leq q(r) \leq 1$ and $q^{\prime}(r) \leq 0$ for all $r \in\left(0, R_{1}\right)$, which proves that $g$ is increasing and $B$ is decreasing. 
6.4 The Chiti comparison result

Sixth step: We prove Lemma 6.2: Here and in the sequel we write short-hand $\lambda_{1}=$ $\lambda_{1}(\Omega)=\lambda_{1}\left(S_{1}\right)$. We introduce a change of variables via $s=C_{n} r^{n}$, where $C_{n}$ is the volume of the $n$-dimensional unit ball. Then by Definition 3.2 we have $u_{1}^{\sharp}(s)=u_{1}^{\star}(r)$ and $z_{1}^{\sharp}(s)=z_{1}(r)$.

Lemma 6.6 For the functions $u_{1}^{\sharp}(s)$ and $z_{1}^{\sharp}(s)$ we have

$$
\begin{aligned}
-\frac{\mathrm{d} u_{1}^{\#}}{\mathrm{~d} s} & \leq \lambda_{1} n^{-2} C_{n}^{-2 / n} s^{n / 2-2} \int_{0}^{s} u_{1}^{\sharp}(w) \mathrm{d} w, \\
-\frac{\mathrm{d} z_{1}^{\sharp}}{\mathrm{d} s} & =\lambda_{1} n^{-2} C_{n}^{-2 / n} s^{n / 2-2} \int_{0}^{s} z_{1}^{\sharp}(w) \mathrm{d} w .
\end{aligned}
$$

Proof We integrate both sides of $-\Delta u_{1}=\lambda_{1} u_{1}$ over the level set $\Omega_{t}:=\{\vec{r} \in \Omega$ : $\left.u_{1}(\vec{r})>t\right\}$ and use Gauss' Divergence Theorem to obtain

$$
\int_{\partial \Omega_{t}}\left|\nabla u_{1}\right| H_{n-1}(\mathrm{~d} r)=\int_{\Omega_{t}} \lambda_{1} u_{1}(\vec{r}) \mathrm{d}^{n} r
$$

where $\partial \Omega_{t}=\left\{\vec{r} \in \Omega: u_{1}(\vec{r})=t\right\}$. Now we define the distribution function $\mu(t)=$ $\left|\Omega_{t}\right|$. Then by Theorem 3.9 we have

$$
\int_{\partial \Omega_{t}}\left|\nabla u_{1}\right| H_{n-1}(\mathrm{~d} r) \geq-n^{2} C_{n}^{2 / n} \frac{\mu(t)^{2-2 / n}}{\mu^{\prime}(t)} .
$$

The left sides of (6.24) and (6.25) are the same, thus

$$
\begin{aligned}
-n^{2} C_{n}^{2 / n} \frac{\mu(t)^{2-2 / n}}{\mu^{\prime}(t)} & \leq \int_{\Omega_{t}} \lambda_{1} u_{1}(\vec{r}) \mathrm{d}^{n} r \\
& =\int_{0}^{\left(\mu(t) / C_{n}\right)^{1 / n}} n C_{n} r^{n-1} \lambda_{1} u_{1}^{\star}(r) \mathrm{d} r .
\end{aligned}
$$

Now we perform the change of variables $r \rightarrow s$ on the right hand side of the above chain of inequalities. We also chose $t$ to be $u_{1}^{\sharp}(s)$. Using the fact that $u_{1}^{\sharp}$ and $\mu$ are essentially inverse functions to one another, this means that $\mu(t)=s$ and $\mu^{\prime}(t)^{-1}=\left(u_{1}^{\sharp}\right)^{\prime}(s)$. The result is (6.22). Equation (6.23) is proven analogously, with equality in each step.

Lemma 6.6 enables us to prove Lemma 6.2. The function $z_{1}^{\sharp}$ is continuous on $\left(0,\left|S_{1}\right|\right)$ and $u_{1}^{\sharp}$ is continuous on $\left(0,\left|\Omega^{\star}\right|\right)$. By the normalization of $u_{1}^{\sharp}$ and $z_{1}^{\sharp}$ and because $S_{1} \subset \Omega^{\star}$ it is clear that either $z_{1}^{\sharp} \geq u_{1}^{\sharp}$ on $\left(0,\left|S_{1}\right|\right)$ or $u_{1}^{\sharp}$ and $z_{1}^{\sharp}$ have at least one intersection on this interval. In the first case there is nothing to prove, simply 
setting $r_{0}=R_{1}$ in Lemma 6.2. In the second case we have to show that there is no intersection of $u_{1}^{\sharp}$ and $z_{1}^{\sharp}$ such that $u_{1}^{\sharp}$ is greater than $z_{1}^{\sharp}$ on the left and smaller on the right. So we assume the contrary, i.e., that there are two points $0 \leq s_{1}<s_{2}<\left|S_{1}\right|$ such that $u_{1}^{\sharp}(s)>z_{1}^{\sharp}(s)$ for $s \in\left(s_{1}, s_{2}\right), u_{1}^{\sharp}\left(s_{2}\right)=z_{1}^{\sharp}\left(s_{2}\right)$ and either $u_{1}^{\sharp}\left(s_{1}\right)=z_{1}^{\sharp}\left(s_{1}\right)$ or $s_{1}=0$. We set

$$
v^{\sharp}(s)= \begin{cases}u_{1}^{\sharp}(s) & \text { on }\left[0, s_{1}\right] \quad \text { if } \int_{0}^{s_{1}} u_{1}^{\sharp}(s) \mathrm{d} s>\int_{0}^{s_{1}} z_{1}^{\sharp}(s) \mathrm{d} s, \\ z_{1}^{\sharp}(s) & \text { on }\left[0, s_{1}\right] \quad \text { if } \int_{0}^{s_{1}} u_{1}^{\sharp}(s) \mathrm{d} s \leq \int_{0}^{s_{1}} z_{1}^{\sharp}(s) \mathrm{d} s, \\ u_{1}^{\sharp}(s) & \text { on }\left[s_{1}, s_{2}\right], \\ z_{1}^{\sharp}(s) & \text { on }\left[s_{2},\left|S_{1}\right|\right] .\end{cases}
$$

Then one can convince oneself that because of (6.22) and (6.23)

$$
-\frac{\mathrm{d} v^{\sharp}}{\mathrm{d} s} \leq \lambda_{1} n^{-2} C_{n}^{-2 / n} s^{n / 2-2} \int_{0}^{s} v^{\sharp}\left(s^{\prime}\right) \mathrm{d} s^{\prime}
$$

for all $s \in\left[0,\left|S_{1}\right|\right]$. Now define the test function $v(r)=v^{\sharp}\left(C_{n} r^{n}\right)$. Using the Rayleigh-Ritz characterization of $\lambda_{1}$, then (6.27) and finally an integration by parts, we get (if $z_{1}$ and $u_{1}$ are not identical)

$$
\begin{aligned}
\lambda_{1} \int_{S_{1}} v^{2}(r) \mathrm{d} x & <\int_{S_{1}}|\nabla v|^{2} \mathrm{~d} x=\int_{0}^{\left|S_{1}\right|}\left(n C_{n} r^{n-1} v^{\sharp^{\prime}}(s)\right)^{2} \mathrm{~d} s \\
& \leq-\int_{0}^{\left|S_{1}\right|} v^{\sharp^{\prime}}(s) \lambda_{1} \int_{0}^{s} v^{\sharp}\left(s^{\prime}\right) \mathrm{d} s^{\prime} \mathrm{d} s \\
& =\lambda_{1} \int_{0}^{\left|S_{1}\right|} v^{\sharp}(s)^{2} \mathrm{~d} s-\lambda_{1}\left[v^{\sharp}(s) \int_{0}^{s} v^{\sharp}\left(s^{\prime}\right) \mathrm{d} s^{\prime}\right]_{0}^{S_{1}} \\
& \leq \lambda_{1} \int_{S_{1}} v^{2}(r) \mathrm{d} x
\end{aligned}
$$

Comparing the first and the last term in the above chain of (in)equalities reveals a contradiction to our assumption that the intersection point $s_{2}$ exists, thus proving Lemma 6.2.

\subsection{Schrödinger operators}

Theorem 6.1 can be extended in several directions. One generalization, which has been considered by Benguria and Linde in [23], is to replace the Laplace operator on the domain $\Omega \subset \mathbb{R}^{n}$ by a Schrödinger operator $H=-\Delta+V$. In this case the question arises which is the most suitable comparison operator for $H$. In analogy to the PPW inequality for the Laplacian, it seems natural to compare the eigenvalues of $H$ to those of another Schrödinger operator $\tilde{H}=-\Delta+\tilde{V}$, which is defined on a ball and has the same lowest eigenvalue as $H$. The potential $\tilde{V}$ should be spherically symmetric and it should reflect some properties of $V$, but it will also have to satisfy 
certain requirements in order for the PPW type estimate to hold. The precise result is stated in Theorem 6.7 below, which can be considered as a natural generalization of Theorem 6.1 to Schrödinger operators.

We assume that $\Omega$ is open and bounded and that $V: \Omega \rightarrow \mathbb{R}^{+}$is a non-negative potential from $L^{1}(\Omega)$. Then we can define the Schrödinger operator $H_{V}=-\Delta+V$ on $\Omega$ in the same way as we did in Section 4.2, i.e., $H_{V}$ is positive and self-adjoint in $L^{2}(\Omega)$ and has purely discrete spectrum. We call $\lambda_{i}(\Omega, V)$ its $i$-th eigenvalue and, as usual, we write $V_{\star}$ for the symmetric increasing rearrangement of $V$.

Theorem 6.7 Let $S_{1} \subset \mathbb{R}^{n}$ be a ball centered at the origin and of radius $R_{1}$ and let $\tilde{V}: S_{1} \rightarrow \mathbb{R}^{+}$be a radially symmetric non-negative potential such that $\tilde{V}(r) \leq V_{\star}(r)$ for all $0 \leq r \leq R_{1}$ and $\lambda_{1}(\Omega, V)=\lambda_{1}\left(S_{1}, \tilde{V}\right)$. If $\tilde{V}(r)$ satisfies the conditions

a) $\tilde{V}(0)=\tilde{V}^{\prime}(0)=0$ and

b) $\tilde{V}^{\prime}(r)$ exists and is increasing and convex,

then

$$
\lambda_{2}(\Omega, V) \leq \lambda_{2}\left(S_{1}, \tilde{V}\right)
$$

If $V$ is such that $V_{\star}$ itself satisfies the conditions a) and b) of the theorem, the best bound is obtained by choosing $\tilde{V}=V_{\star}$ and then adjusting the size of $S_{1}$ such that $\lambda_{1}(\Omega, V)=\lambda_{1}\left(S_{1}, V_{\star}\right)$ holds. (Note that $S_{1} \subset \Omega^{\star}$ by Theorem 4.2). In this case Theorem 6.7 is a typical PPW result and optimal in the sense that equality holds in (6.28) if $\Omega$ is a ball and $V=V_{\star}$. For a general potential $V$ we still get a non-trivial bound on $\lambda_{2}(\Omega, V)$ though it is not sharp anymore.

For further reference we state the following theorem, which is a direct consequence of Theorem 6.7 and Theorem 3.7:

Theorem 6.8 Let $\tilde{V}: \mathbb{R}^{n} \rightarrow \mathbb{R}^{+}$be a radially symmetric positive potential that satisfies the conditions $a$ ) and $b$ ) of Theorem 6.1. Further, assume that $\Omega \subset \mathbb{R}^{n}$ is an open bounded domain and that $S_{1} \subset \mathbb{R}^{n}$ be the open ball (centered at the origin) such that $\lambda_{1}(\Omega, \tilde{V})=\lambda_{1}\left(S_{1}, \tilde{V}\right)$. Then

$$
\lambda_{2}(\Omega, \tilde{V}) \leq \lambda_{2}\left(S_{1}, \tilde{V}\right)
$$

The proof of Theorem 6.7 is similar to the one of Theorem 6.1 and can be found in [23]. One of the main differences occurs in step five (see Section 6.3), since the potential $\tilde{V}(r)$ now appears in the Riccati equation for $p$. It turns out that the conditions a) and b) in Theorem 6.7 are required to establish the monotonicity properties of $q$. A second important difference is that a second eigenfunction of a Schrödinger operator with a spherically symmetric potential can not necessarily be written in the form $u_{2}(r) r_{j} r^{-1}$. It has been shown by Ashbaugh and Benguria [7] that it can be written in this form if $r V(r)$ is convex. On the other hand, the second eigenfunction is radially symmetric (with a spherical nodal surface) if $r V(r)$ is concave. This fact, which is also known as the Baumgartner-Grosse-Martin Inequality [22], is another reason why the conditions a) and b) of Theorem 6.7 are needed. 


\subsection{Gaussian space}

Theorem 6.8 has direct consequences for the eigenvalues of the Laplace operator $-\Delta_{G}$ in Gaussian space, which had been defined in Section 4.3. In this section we write $\lambda_{i}^{-}(\Omega)$ for the $i$-th eigenvalue of $-\Delta_{G}$ on some domain $\Omega$.

Theorem 6.9 Let $\Omega \subset \mathbb{R}^{n}$ be an open bounded domain and assume that $S_{1} \subset \mathbb{R}^{n}$ is a ball, centered at the origin, such that $\lambda_{1}^{-}(\Omega)=\lambda_{1}^{-}\left(S_{1}\right)$. Then

$$
\lambda_{2}^{-}(\Omega) \leq \lambda_{2}^{-}\left(S_{1}\right)
$$

Proof If $\Psi$ is an eigenfunction of $-\Delta_{G}$ on $\Omega$ then $\Psi e^{-r^{2} / 2}$ is an eigenfunction of the Dirichlet-Schrödinger operator $-\Delta+r^{2}$ on $\Omega$, and vice versa. The eigenvalues are related by

$$
\lambda_{i}^{-}(\Omega)=\lambda\left(\Omega, r^{2}-n\right)
$$

where we keep using the notation from Section 6.5. Theorem 6.9 now follows directly from Theorem 6.8, setting $\tilde{V}(r)=r^{2}$.

\subsection{Spaces of constant curvature}

There are generalizations of the Payne-Pólya-Weinberger inequality to spaces of constant curvature. Ashbaugh and Benguria showed in [14] that Theorem 6.1 remains valid if one replaces the Euclidean space $\mathbb{R}^{n}$ by a hemisphere of $\mathbb{S}^{n}$ and 'ball' by 'geodesic ball'. Similar to the Szegö-Weinberger inequality, it is still an open problem to prove a Payne-Pólya-Weinberger result for the whole sphere. Although there seem to be no counterexamples known that rule out such a generalization, the original scheme of proving the PPW inequality is not likely to work. One reason is that numerical studies show the function $g$ to be not monotone on the whole sphere.

For the hyperbolic space, on the other hand, things are settled. Following the general lines of the original proof, Benguria and Linde established in [24] a PPW type inequality that holds in any space of constant negative curvature.

\section{The fundamental gap}

The gap between the first two Dirichlet eigenvalues of the Laplacian, $\lambda_{2}-\lambda_{1}$ and its dependence on the domain $\Omega$, in particular for compact, convex domains in $\mathbb{R}^{n}$, has been considered by several authors. Moreover, many people have considered the more general problem for Schrödinger operators of the form

$$
H=-\Delta+V
$$

on compact, convex domains in $\mathbb{R}^{n}$, with Dirichlet boundary conditions and semiconvex potentials (i.e., potentials $V$ such that $V(x)+c|x|^{2}$ is convex for some $c$ ). Such operators have an increasing sequence of eigenvalues 


$$
\lambda_{1}(\Omega ; V)<\lambda_{2}(\Omega ; V) \leq \lambda_{3}(\Omega ; V) \leq \cdots \rightarrow \infty
$$

For a convex domain $\Omega \subset \mathbb{R}^{n}$, and a convex potential, Singer, et al [92] proved the following lower bounds on the gap $\lambda_{2}-\lambda_{1}$ between the two lowest eigenvalues of the Schrödinger operator $H$ :

$$
\frac{\pi^{2}}{4 d^{2}} \leq \lambda_{2}(\Omega ; V)-\lambda_{1}(\Omega ; V)
$$

where $d$ is the diameter of $\Omega$, i.e., $d=\sup _{x, y \in \Omega}|x-y|$. For the case $V=0$, they also proved the upper bound

$$
\lambda_{2}(\Omega ; 0)-\lambda_{1}(\Omega ; 0) \leq \frac{4 n \pi^{2}}{D^{2}},
$$

where $D$ is the diameter of the largest inscribed ball in $\Omega$.

It was observed by Michael van den Berg [30] that for many exactly solvable cases involving convex domains, the gap $\lambda_{2}-\lambda_{1}$ is bounded from below by $3 \pi^{2} / d^{2}$ (which should be asymptotically achieved by a domain in the shape of a cigar and a constant potential $V$. The value $3 \pi^{2} / d^{2}$ is the actual gap of the Dirichlet Laplacian on an interval of length $d$ in one dimension. This was also independently suggested by Ashbaugh and Benguria [8] and S.-T. Yau [106]. That this bound actually holds for any bounded, convex, domain in $\mathbb{R}^{n}$ and for any semiconvex potential was recently proven by Ben Andrews and Julie Clutterbuck [1]. In fact, we have

Theorem 7.1 (Sharp Lower Bound on the Gap; Andrews and Clutterbuck [1]) Let $\Omega \subset \mathbb{R}^{n}$ be a bounded, convex, domain of diameter $d$, and $V$ a semiconvex potential. The the Dirichlet eigenvalues of the Schrödinger operator H, satisfy,

$$
\lambda_{2}(\Omega ; V)-\lambda_{1}(\Omega ; V) \geq \frac{3 \pi^{2}}{d^{2}} .
$$

Remarks i) In the one dimensional case, for the Schrödinger operator with a more restrictive class of potentials, namely symmetric single well potentials (i.e., centering the interval of length $d$ around 0 and imposing $V(-x)=V(x)$ and $V(x)$ increasing in $[0, d / 2])$ this result was proven by Ashbaugh and Benguria [8]. Later M. Horvath proved a similar result for single well potentials but removing the symmetry condition. In the one dimensional case, for the Schrödinger operator, with a convex potential, this theorem was proven by R. Lavine [66]. Concerning the situation in $\mathbb{R}^{n}$, with $n \geq 2$, in 1986, Yu and Zhong [107] proved the estimate

$$
\lambda_{2}-\lambda_{1} \geq \frac{\pi^{2}}{d^{2}} .
$$

More recently, for $V=0$, Davis [47], and Bañuelos and Kröger [21] proved van den Berg's conjecture for $\Omega \subset \mathbb{R}^{2}$ symmetric with respect to the $x$ and $y$ axes, and convex in both $x$ and $y$. The fundamental gap for the Neumann problem with $V=0$ is just the 
first non trivial Neumann eigenvalue. The sharp lower bound for the first nontrivial Neumann eigenvalue on a convex domain was proven by Payne and Weinberger [85]. For a complete survey on the history of the Fundamental Gap problem up to 2006, see [6].

ii) The proof of Andrews and Clutterbuck relies on two different ingredients, both of which involve analysis of the heat equation. They first introduce the modulus of continuity, $\eta$, of a function $f: \Omega \rightarrow \mathbb{R}$, defined on $\mathbb{R}_{+}$, in such a way that,

$$
|f(y)-f(x)| \leq 2 \eta\left(\frac{|y-x|}{2}\right),
$$

for all $x, y \in \Omega$. It turns out (see [1], and references there in) that if $u$ is any smooth solution of the heat equation with Neumann data on a convex domain $\Omega \subset \mathbb{R}^{n}$ of diameter $d$, then $u(\cdot, t)$ has modulus of continuity $\varphi(\cdot, t)$, where $\varphi$ is the solution to the one-dimensional heat equation on $[-d / 2, d / 2]$ with Neumann boundary data and an initial condition given in terms of the initial condition of the original equation (see [1] for details). Using this method Andrews and Clutterbuck immediately recover the result of [85], which is not strong enough to prove the Fundamental Gap theorem. Thus, they need a second ingredient which is based on a very beautiful generalization of a known technique in this trade, which stems from the log-concavity of the ground state of the Schrödinger Operator for convex potentials (a result that goes back to Brascamp and Lieb [34]). Their generalization is based on the introduction of the so-called modulus of concavity: assume that a one dimensional potential $\tilde{V}$ is even in the interval $[-d / 2, d / 2]$, and assume that the potential $V$ of the Schrödinger operator defined in $\Omega$ is more convex than $\tilde{V}$ in the sense that for any $y \neq x$ in $\Omega$ one has

$$
(\nabla V(y)-\nabla V(x)) \cdot \frac{(y-x)}{|y-x|} \geq 2 \tilde{V}^{\prime}\left(\frac{|y-x|}{2}\right) .
$$

(in their terms, $\tilde{V}^{\prime}$ is thus a modulus of convexity for $V$ ). Then, Andrews and Clutterbuck proved the following improvement on the Brascamp and Lieb result:

Theorem 7.2 Let $\phi_{0}$ be the groundstate of the Schrödinger operator H. If V satisfies (7.2) then, $\phi_{0}$ satisfies the following log-concavity estimate,

$$
\left(\nabla \ln \phi_{0}(y)-\nabla \ln \phi_{0}(x)\right) \cdot \frac{y-x}{|y-x|} \leq\left. 2\left(\ln \tilde{\phi}_{0}\right)^{\prime}\right|_{z=|y-x| / 2},
$$

for every $y \neq x$ in $\Omega$, where $\tilde{\phi}_{0}$ is the ground state of the one dimensional Schrödinger operator $-d^{2} / d x^{2}+\tilde{V}$ in the interval $[-d / 2, d / 2]$ with Dirichlet boundary condition.

Using these two ingredients, Andrews and Clutterbuck prove the following:

Theorem 7.3 If $V$ and $\tilde{V}$ are related by (7.2), then the fundamental gap for the Schrödinger operator $H=-\Delta+V$, on a bounded, convex domain $\Omega$ is bounded below by the fundamental gap of the one dimensional operator $-d^{2} / d x^{2}+\tilde{V}$ on the interval $[-d / 2, d / 2]$. 
In particular, if the potential $V$ is convex, one can choose $\tilde{V}=0$ and van den Berg's Conjecture follows.

Moreover, Andrews and Clutterbuck prove the following extension:

Theorem 7.4 If $V$ is semiconvex, then, the fundamental gap satisfies the bound,

$$
\lambda_{2}-\lambda_{1} \geq \frac{3 \pi^{2}}{d^{2}} .
$$

For the proofs of these results, references and extensions see [1].

\section{An isoperimetric inequality for ovals in the plane}

The next problem we will consider is a conjectured isoperimetric inequality for closed, smooth curves in the plane. It has attracted considerable attention in the literature during the last decade (see, e.g., [37,48,49,56,57]), and it has many interesting connections in geometry and physics. In particular, Benguria and Loss [26] have shown a connection between this problem and a special case of the Lieb-Thirring inequalities $[68,71]$, inequalities which play a fundamental role in Lieb and Thirring's proof of the stability of matter (see, in particular, [70] and the review article [67]).

Denote by $C$ a closed curve in the plane, of length $2 \pi$, with positive curvature $\kappa$, and let

$$
H(C) \equiv-\frac{d^{2}}{d s^{2}}+\kappa^{2}
$$

acting on $L^{2}(C)$ with periodic boundary conditions. Let $\lambda_{1}(C)$ denote the lowest eigenvalue of $H(C)$. Certainly, $\lambda_{1}(C)$ depends on the geometry of the curve $C$. It has been conjectured that

$$
\lambda_{1}(C) \geq 1
$$

with equality if and only if $C$ belongs to a one-parameter family of ovals which include the circle (in fact the one-parameter family of curves is characterized by a curvature given by $\left.\kappa(s)=1 /\left(a^{2} \cos ^{2}(s)+a^{-2} \sin ^{2}(s)\right)[26]\right)$. It is a simple matter to see that if $C$ is a circle of length $2 \pi$, the lowest eigenvalue of $H(C)$ is precisely 1 . The fact that there is degeneracy of the conjectured minimizers makes the problem much harder.

The conjecture (8.2) is still open. Concerning (nonoptimal) lower bounds, Benguria and Loss proved [26]

$$
\lambda_{1}(C) \geq 1 / 2
$$

and more recently Linde [72] found the best lower bound to date,

$$
\lambda_{1}(C)>\left(1+\frac{\pi}{\pi+8}\right)^{-2} \approx 0.60847
$$


Even though this is the best bound to date, it is still some distance from the conjectured optimal value 1 .

Burchard and Thomas [38] have shown that the ovals that give $\lambda_{1}(C)=1$, alluded to before, minimize $\lambda_{1}(C)$ at least locally, i.e., there is no small variation around these curves that reduces $\lambda_{1}(C)$. This is an important indication that the conjecture (8.2) is true, though, of course, it is not enough to prove it and thus it remains open.

In recent years several authors have obtained isoperimetric inequalities for the lowest eigenvalues of a variant of $H(C)$, and we give a short summary of the main results in the sequel. Consider the Schrödinger operator

$$
H_{g}(C) \equiv-\frac{d^{2}}{d s^{2}}+g \kappa^{2}
$$

defined on $L^{2}(C)$ with periodic boundary conditions. As before, $C$ denotes a closed curve in $\mathbb{R}^{2}$ with positive curvature $\kappa$, and length $2 \pi$. Here, $s$ denotes arclength. If $g<0$, the lowest eigenvalue of $H_{g}(C)$, say $\lambda_{1}(g, C)$, is uniquely maximized when $C$ is a circle [48]. When $g=-1$, the second eigenvalue, $\lambda_{2}(-1, C)$, is uniquely maximized when $C$ is a circle [57]. If $0<g \leq 1 / 4, \lambda_{1}(g, C)$ is uniquely minimized when $C$ is a circle [49]. It is an open problem to determine the curve $C$ that minimizes $\lambda_{1}(g, C)$ in the cases, $1 / 4<g \leq 1$, and $g<0, g \neq-1$. If $g>1$ the circle is not a minimizer for $\lambda_{1}(g, C)$ (see, e.g., $[49,56]$ for more details on the subject).

Remark Very recently, Bernstein and Breiner [31] have introduced a new twist on this problem (i.e., on the conjectured isoperimetric inequality for ovals on the plane). Bernstein and Breiner have considered the Catenoid

$$
\text { Cat }=\left\{x_{1}^{2}+x_{2}^{2}=\cosh ^{2}\left(x_{3}\right)\right\} \subset \mathbb{R}^{3},
$$

which is a minimal surface of revolution introduced by Euler in 1744 . They showed how the conjectured inequality of Benguria and Loss could be used to prove that subsets of the Catenoid minimize area within a geometric natural class of minimal annuli.

\section{Fourth order differential operator}

In continuum mechanics, the vibrations of more rigid objects, like plates, rods, etc., are governed by wave equations involving higher order operators on the spatial variables. The normal modes of oscillations of these equations give rise to an eigenvalue problem associated to fourth order operators. There are isoperimetric inequalities for these eigenvalues, which are analogous to the ones that we have been discussing for vibrating membranes. In this section we will briefly review three of these isoperimetric inequalities, arising in connection to i) the vibrations of the clamped plate, ii) the buckling problem, and iii) the vibrations of the free vibrating plate. In connection to fourth order operators, there is also a vast literature (in particular in the last few years) involving universal inequalities for the eigenvalues of these spectral problems. We will not discuss these universal inequalities here. 


\subsection{The clamped plate}

Consider a bounded, smooth domain $\Omega \subset \mathbb{R}^{2}$. The eigenvalue problem that determines the eigenfrequencies of a clamped plate is given by

$$
\Delta^{2} u=\Gamma u, \quad \text { in } \Omega
$$

together with the clamped boundary conditions,

$$
u=|\nabla u|=0, \quad \text { in } \partial \Omega
$$

(the eigenfrequencies are proportional to the square root of the eigenvalues). The boundary value problem $(9.1),(9.2)$ has a countable sequence of eigenvalues

$$
0<\Gamma_{1}(\Omega) \leq \Gamma_{2}(\Omega) \leq \ldots
$$

and $\Gamma_{n}(\Omega) \rightarrow \infty$ as $n \rightarrow \infty$. Because we are dealing with the operator $\Delta^{2}$ (and not just the Laplacian) two nasty things may occur:

i) The principal eigenfunction $u_{1}$ of the boundary value problem defined by (9.1) and (9.2) is not necessarily of one sign (say positive).

ii) The lowest eigenvalue $\Gamma_{1}$ for the clamped plate may be degenerate.

There is an extensive literature on these two facts (see, in particular the Bibliographical Remarks i), ii) and iii) at the end of this section). These two facts make it impossible to use the standard techniques that we have discussed in previous sections in connection with the proof of isoperimetric inequalities for the Laplacian and Schrödinger operators.

9.2 Rayleigh's conjecture for the clamped plate

In the first edition of his book The Theory of Sound, Lord Rayleigh conjectured [88] that

$$
\Gamma_{1}(\Omega) \geq \Gamma_{1}\left(\Omega^{*}\right)
$$

where $\Gamma_{1}(\Omega)$ denotes the first eigenvalue for the vibrations of a clamped plate, and $\Omega^{*}$ is a disk with the same area as $\Omega$.

In 1950, G. Szegö [95] (see also [96], and the Erratum [98]) proved Rayleigh's conjecture for the clamped plate under the assumption that $u_{1}$ is of one sign (assumption which we now know does not always hold).

A significant step towards the proof of Rayleigh's conjecture was done by G. Talenti [99] in 1981. Finally, in 1995, the conjecture was proven by N.S. Nadirashvili [79]. The analog of Rayleigh's conjecture for the clamped plate in three dimensions was proven by M.S. Ashbaugh and R.D. Benguria [13] (see also, [17]). The analog of Rayleigh's conjecture for the clamped plate in dimensions larger than 3 (i.e., $n \geq 4$ ) is still an open problem. Although not sharp, the best results to date for $n \geq 4$ have been obtained by M. S. Ashbaugh and R. S. Laugesen, [18]. 
The proof of Rayleigh's conjecture for the clamped plate, i.e., the proof of (9.3) is based on several steps. The first step, as usual, is the variational characterization of $\Gamma_{1}(\Omega)$. In the second step, taking into account that the ground state, say $u$, of (9.1) and (9.2) is not necessarily positive, one defines the sets $\Omega_{+}=\left\{x \mid u_{+}>0\right\}$ and $\Omega_{-}=\left\{x \mid u_{-}>0\right\}$, where $u_{+}=\max (u, 0)$ and $u_{-}=\max (-u, 0)$ are the positive and negative parts of $u$, respectively. The third step is to consider the positive and negative parts of $\Delta u$, in other words we write $\Delta u=(\Delta u)_{+}-(\Delta u)_{-}$. Then, one considers the rearrangement,

$$
g(s)=(\Delta u)_{+}^{*}(s)-(\Delta u)_{-}^{*}(\mu(\Omega)-s),
$$

where $s=C_{n}|x|^{n}$, and $C_{n}$ is the volume of the unit ball in $n$ dimensions (here $n=2$ or 3 , as we mentioned above), and $\mu(\Omega)$ is the volume of $\Omega$. The next step is to consider the solutions, $v$ and $w$ of some Dirichlet problem in the balls $\Omega_{+}^{*}$ and $\Omega_{-}^{*}$ respectively. Then one uses a comparison theorem of Talenti [99], namely $u_{+}^{*} \leq v$ in $\Omega_{+}^{*}$ and $u_{-}^{*} \leq w$ in $\Omega_{-}^{*}$, respectively. The functions $v$ and $w$ can be found explicitly in terms of modified Bessel functions. The final step is to prove the necessary monotonicity properties of these functions $v$, and $w$. We refer the reader to [13], for details.

\subsection{Rayleigh's conjecture for the buckling of a clamped plate}

The buckling eigenvalues for the clamped plate for a domain $\Omega$ in $\mathbb{R}^{2}$, correspond to the eigenvalues of the following boundary value problem,

$$
-\Delta^{2} u=\Lambda \Delta u, \quad \text { in } \Omega
$$

together with the clamped boundary conditions,

$$
u=|\nabla u|=0, \quad \text { in } \partial \Omega
$$

The lowest eigenvalue, $\Lambda_{1}$ say, is related to the minimum uniform load applied in the boundary of the plate necessary to buckle it. There is a conjecture of L. Payne [81], regarding the isoperimetric behavior of $\Lambda_{1}$, namely,

$$
\Lambda_{1}(\Omega) \geq \Lambda_{1}\left(\Omega^{*}\right)
$$

To prove this conjecture is still an open problem. For details see, e.g., [81,18], and the review articles [82,3]. Recently, Antunes [2] has checked numerically Payne's conjecture for a large class of domains (mainly families of triangles or other simple polygons). Also, in [2] Antunes has studied the validity of other eigenvalue inequalities (mainly relating the $\Lambda_{1}$ with different Dirichlet eigenvalues for the same domain $\Omega$.

\subsection{The fundamental tones of free plates}

The analog of the Szegö-Weinberger problem for the "free vibrating plate" has been recently considered by L. Chasman, in her Ph.D. thesis [39] (see also [40,41]). As discussed in [39], the fundamental tone for that problem, say $\omega_{1}(\Omega)$, corresponds to the first nontrivial eigenvalue for the boundary value problem 


\section{$\Delta \Delta u-\tau \Delta u=\omega u$,}

in a bounded region $\Omega$ in the $d$ dimensional Euclidean Space, with some natural boundary conditions, where $\tau$ is a positive constant. In fact $\omega_{1}(\Omega)$ is the fundamental tone of a free vibrating plate with tension (physically $\tau$ represents the ratio of the lateral tension to the flexural rigidity). In $[39,40]$, Chasman proves the following isoperimetric inequality,

$$
\omega_{1}(\Omega) \leq \omega_{1}\left(\Omega^{*}\right)
$$

where $\Omega^{*}$ is a ball of the same volume as $\Omega$ (here, equality is attained if and only if $\Omega$ is a ball). This result is the natural generalization of the corresponding SzegöWeinberger result for the fundamental tone of the free vibrating membrane. Here, we will not discuss the exact boundary conditions appropriate for this problem (we refer the reader to [39] for details). In fact, the appropriate "free" boundary conditions are essentially obtained as some transversality conditions of the Direct Calculus of Variations for this problem. In [39], Chasman first derives the equivalent of the classical result of F. Pöckels for the membrane problem in this case, i.e., the existence of a discrete sequence of positive eigenvalues accumulating at infinity. Then, she carefully discusses the free boundary conditions both, for smooth domains, and for domains with corners (in fact she considers as specific examples the rectangle and the ball, and, moreover the analog one dimensional problem). Then, universal upper and lower bounds are derived for $\omega_{1}$ in terms of $\tau$. As for the proof of the isoperimetric inequality for $\omega_{1}$, she uses a similar path as the one used in the proof of the Szegö-Weinberger inequality Since in the present situation the operator is more involved, this task is not easy. She starts by carefully analyzing the necessary monotonicity properties of the Bessel (and modified) Bessel functions that naturally appear in the solution for the ( $d$-degenerate) eigenfunctions corresponding to the fundamental tone, $\omega_{1}$, of the ball. Then, the Weinberger strategy takes us through the standard road: use the variational characterization of $\omega_{1}(\Omega)$ in terms of $d$ different trial functions (given as usual as a radial function $\rho$ times the angular part $x_{i} / r$, for $i=1, \ldots, d$ ) and averaging, to get a rotational invariant, variational upper bound on $\omega_{1}$. As usual, a Brower's fixed point theorem is needed to insure the orthogonality of these trial functions to the constants. Then, one chooses the right expression for the variational function $\rho$ guided by the expressions of the eigenfunctions associated to the fundamental tone of the ball. As in the proof of many of the previous isoperimetric inequalities, Chasman has to prove monotonicity properties of $\rho$ (chosen as above) and of the expressions involving $\rho$ and higher derivatives that appear in the bound obtained after the averaging procedure in the previous section. Finally, rearrangements and symmetrization arguments are used to conclude the proof of the isoperimetric result (see [39] for details).

\subsection{Bibliographical remarks}

i) There is a recent, very interesting article on the sign of the principal eigenfunction of the clamped plate by Guido Sweers, When is the first eigenfunction for the clamped plate equation of fixed sign, USA-Chile Workshop on Nonlinear Analysis, Electronic J. Diff. Eqns., Conf. 06, 2001, pp. 285-296, available on the web at 
http://ejde.math.swt.edu/conf-proc/06/s3/sweers.pdf], where the author reviews the status of this problem and the literature up to 2001

ii) For general properties of the spectral properties of fourth order operators the reader may want to see: Mark P. Owen, Topics in the Spectral Theory of 4th order Elliptic Differential Equations, Ph.D. Thesis, University of London, 1996. Available on the Web at http://www.ma.hw.ac.uk/ mowen/research/thesis/thesis.ps

iii) Concerning the two problems mentioned in the introduction of Section 9, the reader may want to check the following references: R. J. Duffin, On a question of Hadamard concerning super-biharmonic functions, J. Math. Phys. 27, 253-258 (1949); R. J. Duffin, D. H. Shaffer, On the modes of vibration of a ring-shaped plate, Bull. AMS 58, 652 (1952); C.V. Coffman, R. J. Duffin, D. H. Shaffer, The fundamental mode of vibration of a clamped annular plate is not of one sign, in Constructive approaches to mathematical models (Proc. Conf. in honor of R. Duffin, Pittsburgh, PA, 1978), pp. 267-277, Academic Press, NY (1979); C.V. Coffman, R. J. Duffin, On the fundamental eigenfunctions of a clamped punctured disk, Adv. in Appl. Math. 13, 142-151 (1992).

Acknowledgments We would like to thank the anonymous referee for many helpful suggestions and remarks. The work of RB and BL has been partially supported by Iniciativa Científica Milenio, ICM (CHILE), project P07-027-F. The work of RB has also been supported by FONDECYT (Chile) Project 1100679.

Open Access This article is distributed under the terms of the Creative Commons Attribution License which permits any use, distribution and reproduction in any medium, provided the original author(s) and source are credited.

\section{Appendix}

10.1 The layer-cake formula

Theorem 10.1 Let $v$ be a measure on the Borel sets of $\mathbb{R}^{+}$such that $\Phi(t):=v([0, t))$ is finite for every $t>0$. Let further $(\Omega, \Sigma, m)$ be a measure space and $v$ a non-negative measurable function on $\Omega$. Then

$$
\int_{\Omega} \Phi(v(x)) m(\mathrm{~d} x)=\int_{0}^{\infty} m(\{x \in \Omega: v(x)>t\}) v(\mathrm{~d} t) .
$$

In particular, if $m$ is the Dirac measure at some point $x \in \mathbb{R}^{n}$ and $v(\mathrm{~d} t)=\mathrm{d} t$ then (10.1) takes the form

$$
v(x)=\int_{0}^{\infty} \chi\{y \in \Omega: v(y)>t\}(x) \mathrm{d} t .
$$


Proof Since $m(\{x \in \Omega: v(x)>t\})=\int_{\Omega} \chi_{\{v>t\}}(x) m(\mathrm{~d} x)$ we have, using Fubini's theorem,

$$
\int_{0}^{\infty} m(\{x \in \Omega: v(x)>t\}) v(\mathrm{~d} t)=\int_{\Omega}\left(\int_{0}^{\infty} \chi_{\{v>t\}}(x) v(\mathrm{~d} t)\right) m(\mathrm{~d} x) .
$$

Theorem 10.1 follows from observing that

$$
\int_{0}^{\infty} \chi_{\{v>t\}}(x) v(\mathrm{~d} t)=\int_{0}^{v(x)} v(\mathrm{~d} t)=\Phi(v(x)) .
$$

10.2 A consequence of the Brouwer fixed-point theorem

Theorem 10.2 (Brouwer's fixed-point theorem) Let $B \subset \mathbb{R}^{n}$ be the unit ball for $n \geq 0$. If $f: B \rightarrow B$ is continuous then $f$ has a fixed point, i.e., there is some $x \in B$ such that $f(x)=x$.

The proof appears in many books on topology, e.g., in [78]. Brouwer's theorem can be applied to establish the following result:

Theorem 10.3 Let $B \subset \mathbb{R}^{n}(n \geq 2)$ be a closed ball and $\vec{b}(\vec{r})$ a continuous map from $B$ to $\mathbb{R}^{n}$. If $\vec{b}$ points strictly outwards at every point of $\partial B$, i.e., if $\vec{b}(\vec{r}) \cdot \vec{r}>0$ for every $\vec{r} \in \partial B$, then $\vec{b}$ has a zero in $B$.

Proof Without losing generality we can assume that $B$ is the unit ball centered at the origin. Since $\vec{b}$ is continuous and $\vec{b}(\vec{r}) \cdot \vec{r}>0$ on $\partial B$, there are two constants $0<r_{0}<1$ and $p>0$ such that $\vec{b}(\vec{r}) \cdot \vec{r}>p$ for every $\vec{r}$ with $r_{0}<|\vec{r}| \leq 1$. We show that there is a constant $c>0$ such that

$$
|-c \vec{b}(\vec{r})+\vec{r}|<1
$$

for all $\vec{r} \in B$ : In fact, for all $\vec{r}$ with $|\vec{r}| \leq r_{0}$ the constant $c$ can be any positive number below ( $\left.\sup _{\vec{r} \in B}|\vec{b}(\vec{r})|\right)^{-1}\left(1-r_{0}\right)$. The supremum exists because $|\vec{b}|$ is continuously defined on a compact set and therefore bounded. On the other hand, for all $\vec{r} \in B$ with $|\vec{r}|>r_{0}$ we have

$$
\begin{aligned}
|-c \vec{b}(\vec{r})+\vec{r}|^{2} & =c^{2}|\vec{b}(\vec{r})|^{2}-2 c \vec{b}(\vec{r}) \cdot \vec{r}+|\vec{r}|^{2} \\
& \leq c^{2} \sup _{\vec{r} \in B}|\vec{b}|^{2}-2 c p+1,
\end{aligned}
$$

which is also smaller than one if one chooses $c>0$ sufficiently small. Now set

$$
\vec{g}(\vec{r})=-c \vec{b}(\vec{r})+\vec{r} \text { for } \vec{r} \in B
$$

Then $\vec{g}$ is a continuous mapping from $B$ to $B$ and by Theorem 10.2 it has some fixed point $\vec{r}_{1} \in B$, i.e., $\vec{g}\left(\vec{r}_{1}\right)=\vec{r}_{1}$ and $\vec{b}\left(\vec{r}_{1}\right)=0$. 


\section{References}

1. Andrews, B., Clutterbuck, J.: Proof of the fundamental gap conjecture. J. Am. Math. Soc. 24, 899916 (2011)

2. Antunes, P.R.S.: On the buckling eigenvalue Problem. J. Phys. A Math. Theor. 44, 215205(13) (2011)

3. Ashbaugh, M.S.: Isoperimetric and universal inequalities for eigenvalues. In: Davies B., Safarov Yu. (eds.) Spectral Theory and Geometry (Edinburgh, 1998), E. London Math. Soc. Lecture Notes, vol. 273, pp. 95-139. Cambridge University Press, Cambridge (1999)

4. Ashbaugh, M.S.: Open problems on eigenvalues of the Laplacian. In: Rassias, Th.M., Srivastava, H.M. (eds.) Analytic and Geometric Inequalities and Applications, Mathematics and Its Applications, 478, pp. 13-28. Kluwer Academic Publishers, Dordrecht (1999) [see also M. S. Ashbaugh contribution (pp. 2-10) to ESI-Workshop on Geometrical Aspects of Spectral Theory (Matrei, Austria, 1999), L. Friedlander and T. HoffmannOstenhof, editors, 33 pp., available electronically at http://www.esi.ac. at/ESIPreprints.html, preprint no. 768]

5. Ashbaugh M.S.: The universal eigenvalue bounds of Payne-Pólya-Weinberger, Hile-Protter, and H. C. Yang, In: Spectral and Inverse Spectral Theory (Goa, 2000). Proc. Indian Acad. Sci. Math. Sci., vol. 112, 3-30 (2002)

6. Ashbaugh M.S.: The Fundamental Gap (2006) http://www.aimath.org/WWN/loweigenvalues/

7. Ashbaugh, M.S., Benguria, R.D.: Log-concavity of the ground state of Schrödinger operators: a new proof of the Baumgartner-Grosse-Martin inequality. Phys. Lett. A 131, 273-276 (1988)

8. Ashbaugh, M.S., Benguria, R.D.: Optimal lower bound for the gap between the first two eigenvalues of one-dimensional Schrödinger operators with symmetric single well potentials. Proc. Am. Math. Soc. 105, 419-424 (1989)

9. Ashbaugh, M.S., Benguria, R.D.: Proof of the Payne-Pólya-Weinberger conjecture. Bull. Am. Math. Soc. 25, 19-29 (1991)

10. Ashbaugh, M.S., Benguria, R.D.: A sharp bound for the ratio of the first two eigenvalues of Dirichlet Laplacians and extensions. Ann. Math. 135, 601-628 (1992)

11. Ashbaugh, M.S., Benguria, R.D.: A second proof of the Payne-Pólya-Weinberger conjecture. Commun. Math. Phys. 147, 181-190 (1992)

12. Ashbaugh, M.S., Benguria, R.D.: Sharp upper bound to the first nonzero Neumann eigenvalue for bounded domains in spaces of constant curvature. J. Lond. Math. Soc. 52(2), 402-416 (1995)

13. Ashbaugh, M.S., Benguria, R.D.: On Rayleigh's conjecture for the clamped plate and its generalization to three dimensions. Duke Math. J. 78, 1-17 (1995)

14. Ashbaugh, M.S., Benguria, R.D.: A sharp bound for the ratio of the first two Dirichlet eigenvalues of a domain in a hemisphere of $\mathbb{S}^{n}$. Trans. Am. Math. Soc. 353, 1055-1087 (2001)

15. Ashbaugh, M.S., Benguria, R.D.: Isoperimetric inequalities for eigenvalues of the Laplacian. In: Gesztesy, F., Deift, P., Galvez, C., Perry, P., Schlag, W. (eds.) Spectral Theory and Mathematical Physics: A Festschrift in Honor of Barry Simon's 60th Birthday, Proceedings of Symposia in Pure Mathematics, vol. 76, Part 1, pp. 105-139. Amer. Math. Soc., Providence, RI (2007)

16. Ashbaugh, M.S., Benguria, R.D.: El problema de la Reina Dido: Panorama sobre los problemas de la isoperimetría, Joven Matemático, vol. 1, 3-8 (2010). [The original English version of this essay, Dido and Isoperimetry, can be found on the web page of the International Conference on the isoperimetric problem of Queen Dido and its mathematical ramifications, that was held in Carthage, Tunisia, May 24-29, 2010; see, http://math.arizona.edu/ dido/didon.html; a french translation, by Jacqueline Fleckinger-Pellé, can be found in the same web page]

17. Ashbaugh, M.S., Benguria, R.D., Laugesen R.S.: Inequalities for the first eigenvalues of the clamped plate and buckling problems. in General inequalities 7, (Oberwolfach, 1995), pp. 95-110. Internat. Ser. Numer. Math., vol. 123. Birkhäuser, Basel (1997)

18. Ashbaugh, M.S., Laugesen, R.S.: Fundamental tones and buckling loads of clamped plates. Ann. Scuola Norm. Sup. Pisa Cl. Sci. 23, 383-402 (1996)

19. Baernstein II, A.: A unified approach to symmetrization, In: Partial Differential Equations of Elliptic Type, Cortona, 1992, Sympos. Math., vol. XXXV, pp. 47-91. Cambridge University Press, Cambridge, UK (1994)

20. Bandle, C.: Isoperimetric inequalities and applications, Pitman monographs and studies in mathematics, vol. 7. Pitman, Boston (1980)

21. Bañuelos, R., Kröger, P.: Gradient estimates for the ground state Schrödinger eigenfunction and applications. Commun. Math. Phys. 224, 545-550 (2001) 
22. Baumgartner, B., Grosse, H., Martin, A.: The Laplacian of the potential and the order of energy levels. Phys. Lett. B 146, 363-366 (1984)

23. Benguria, R.D., Linde, H.: A second eigenvalue bound for the Dirichlet Schrödinger operator. Commun. Math. Phys. 267, 741-755 (2006)

24. Benguria, R.D., Linde, H.: A second eigenvalue bound for the Dirichlet Laplacian in hyperbolic space. Duke Math. J. 140, 245-279 (2007)

25. Benguria, R.D., Linde, H.: Isoperimetric inequalities for eigenvalues of the Laplace operator. In: Villegas-Blas, C. (ed.) Fourth summer school in analysis and mathematical physics: topics in spectral theory and quantum mechanics. Contemporary Mathematics (AMS), vol. 476, pp. 1-40 (2008)

26. Benguria, R.D., Loss, M.: Connection between the Lieb-Thirring conjecture for Schrödinger operators and an isoperimetric problem for ovals on the plane. In: Conca, C., Manásevich, R., Uhlmann, G., Vogelius, M.S. (eds.) Partial Differential Equations and Inverse Problems. Contemp. Math., vol. 362, pp. 53-61. Amer. Math. Soc., Providence, R.I. (2004)

27. Bérard, P.: Transplantation et isospectralité I. Math. Ann. 292, 547-559 (1992)

28. Bérard, P.: Transplantation et isospectralité II. J. Lond. Math. Soc. 48, 565-576 (1993)

29. Bérard, P.: Domaines plans isospectraux a la Gordon-Web-Wolpert: une preuve elementaire. Afr. Math. 1, 135-146 (1993)

30. van den Berg, M.: On condensation in the free-boson gas and the spectrum of the Laplacian. J. Stat. Phys. 31, 623-637 (1983)

31. Bernstein, J., Breiner, C.: A variational characterization of the catenoid. (2010) (preprint)

32. Birman, M.S., Solomjak, M.Z.: Spectral theory of self-adjoint operators in Hilbert Space. D. Reidel Publishing Company, Dordrecht (1987)

33. Borell, C.: The Brunn-Minkowski inequality in Gauss space. Invent. Math. 30, 207-211 (1975)

34. Brascamp, J., Lieb, E.H.: On extensions of the Brunn-Minkowski and Prékopa-Leindler theorems, including inequalities for log concave functions, and with an application to the diffusion equation. J. Funct. Anal. 22, 366-389 (1976)

35. Brock, F., Solynin, A.Y.: An approach to symmetrization via polarization. Trans. Am. Math. Soc. 352, 1759-1796 (2000)

36. Brooks, R.: Constructing isospectral manifolds. Am. Math. Mon. 95, 823-839 (1988)

37. Burchard, A., Thomas, L.E.: On the Cauchy problem for a dynamical Euler's elastica. Commun. Partial Differ. Equ. 28, 271-300 (2003)

38. Burchard, A., Thomas, L.E.: On an isoperimetric inequality for a Schrödinger operator depending on the curvature of a loop. J. Geom. Anal. 15, 543-563 (2005)

39. Chasman, L.M.: Isoperimetric problem for eigenvalues of free plates. Ph. D thesis, University of Illinois at Urbana-Champaign (2009)

40. Chasman, L.M.: An isoperimetric inequality for fundamental tones of free plates. Commun. Math. Phys. 303, 421-429 (2011)

41. Chasman, L.M.: Vibrational modes of circular free plates under tension. Appl. Anal. 90, 18771895 (2011)

42. Chavel, I.: Eigenvalues in Riemannian geometry. Academic Press, NY (1984)

43. Courant, R., Hilbert, D.: Methods of Mathematical Physics, vol. 1. Interscience Publishers, New York (1953)

44. Daners, D.: A Faber-Krahn inequality for Robin problems in any space dimension. Math. Ann. 335, 767-785 (2006)

45. Davies, E.B.: Heat kernels and spectral theory, paperback edn. Cambridge University Press, Cambridge (1990)

46. Davies, E.B.: Spectral theory and differential operators. Cambridge University Press, Cambridge (1996)

47. Davis, B.: On the spectral gap for fixed membranes. Ark. Mat. 39, 65-74 (2001)

48. Duclos, P., Exner, P.: Curvature-induced bound states in quantum waveguides in two and three dimensions. Rev. Math. Phys. 7, 73-102 (1995)

49. Exner, P., Harrell, E.M., Loss, M.: Optimal eigenvalues for some Laplacians and Schrödinger operators depending on curvature. In: Dittrich, J., Exner, P., Tater, M. (eds.) Mathematical Results in Quantum Mechanics (Prague, 1998). Oper. Theory Adv. Appl., vol. 108, pp. 47-58 (1999)

50. Faber, G.: Beweis, dass unter allen homogenen Membranen von gleicher Fläche und gleicher Spannung die kreisförmige den tiefsten Grundton gibt. In: Sitzungberichte der mathematischphysikalischen Klasse der Bayerischen Akademie der Wissenschaften zu München Jahrgang, pp. 169-172 (1923) 
51. Federer, H.: Geometric Measure Theory. Springer Verlag, New York (1969)

52. Geisinger, L., Weidl, T.: Universal bounds for traces of the Dirichlet Laplace operator. J. Lond. Math. Soc. 82, 395-419 (2010)

53. Giraud, O., Thas, K.: Hearing shapes of drums: Mathematical and physical aspects of isospectrality. Rev. Mod. Phys. 82, 2213-2255 (2010)

54. Gordon, C., Webb, D., Wolpert, S.: Isospectral plane domains and surfaces via Riemannian orbifolds. Invent. Math. 110, 1-22 (1992)

55. Hardy, G.H., Littlewood, J.E., Pólya, G.: Inequalities. Cambridge Univ. Press, Cambridge, UK (1964)

56. Harrell, E.M.: Gap estimates for Schrödinger operators depending on curvature talk delivered at the 2002 UAB International Conference on Differential Equations and Mathematical Physics. Available electronically at http://www.math.gatech.edu/ harrell/

57. Harrell, E.M., Loss, M.: On the Laplace operator penalized by mean curvature. Commun. Math. Phys. 195, 643-650 (1998)

58. Harrell, E.M., Hermi, L.: Differential inequalities for Riesz means and Weyl type bounds for eigenvalues. J. Funct. Anal. 254, 3173-3191 (2008)

59. Heath, T.L.: A History of Greek Mathematics. vol. 2. The Clarendon Press, Oxford (1921)

60. Henrot, A.: Extremum Problems for Eigenvalues of Elliptic Operators. Collection Frontiers in Mathematics, Birkhauser (2006)

61. Kac M.: On some connections between probability theory and differential and integral equations. In: Neyman, J. (ed.) Proceedings of the Second Berkeley Symposium on Mathematical Statistics and Probability. University of California Press, Berkeley, CA, pp. 189-215 (1951)

62. Kac, M.: Can one hear the shape of a drum?. Am. Math. Mon. 73, 1-23 (1966)

63. Kornhauser, E.T., Stakgold, I.: A variational theorem for $\nabla^{2} u+\lambda u=0$ and its applications. J. Math. Phys. 31, 45-54 (1952)

64. Krahn, E.: Über eine von Rayleigh formulierte Minimaleigenschaft des Kreises. Math. Ann. 94, 97$100(1925)$

65. Krahn, E. :Über Minimaleigenschaften der Kugel in drei und mehr Dimensionen, Acta Comm. Univ. Tartu (Dorpat), vol. A9, 1-44 (1926) [English translation: Minimal properties of the sphere in three and more dimensions. In: Edgar Krahn 1894-1961: A Centenary Volume, Ü. Lumiste and J. Peetre, editors, IOS Press, Amsterdam, The Netherlands, pp. 139-174 (1994)]

66. Lavine, R.: The eignevalue gap for one-dimensional convex potentials. Proc. Am. Math. Soc. 121, 815-821 (1994)

67. Lieb, E.H.: The stability of matter Rev. Mod. Phys. 48, 553-569 (1976)

68. Lieb, E.H.: Lieb-Thirring inequalities. In: Encyclopaedia of Mathematics, Suppl. II, Kluwer, Dordrecht, pp. 311-312 (2000)

69. Lieb, E.H., Loss, M.: Analysis, Graduate Studies in Mathematics, vol. 14. American Mathematical Society, Providence, RI (1997)

70. Lieb, E.H., Thirring, W.: Bounds for the kinetic energy of fermions which proves the stability of matter. Phys. Rev. Lett. 35, 687-689 (1975) (Errata: PRL 35, 1116 (1975))

71. Lieb, E.H., Thirring, W.: Inequalities for the moments of the eigenvalues of the Schrödinger hamiltonian and their relation to Sobolev inequalities. In: Lieb, E.H., Simon, B., Wightman, A.S. Studies in Mathematical Physics, Essays in Honor of Valentine Bargmann, Princeton University Press, Princeton (1986)

72. Linde, H.: A lower bound for the ground state energy of a Schrödinger operator on a loop. Proc. Am. Math. Soc. 134, 3629-3635 (2006)

73. McHale, K.P.: Eigenvalues of the Laplacian, “Can you Hear the Shape of a Drum?”, Master's Project. Mathematics Department University of Missouri, Columbia, MO (1994)

74. McKean, H.P., Singer, I.M.: Curvature and the eigenvalues of the Laplacian. J. Differ. Geom. 1, 662670 (1967)

75. Maz'ja V.G. : Sobolev spaces, Springer Series in Soviet Mathematics. Springer-Verlag, Berlin (1985) (Translated from the Russian by T.O. Shaposhnikova)

76. Melrose, R.B.: The inverse spectral problem for planar domains. In: Proceedings of the Centre for Mathematics and its Applications, Australian National University, vol. 34 (1996)

77. Milnor, J.: Eigenvalues of the Laplace operator on certain manifolds. Proc. Nat. Acad. Sci. 51, 542 (1964)

78. Munkres, J.R.: Topology, A first course. Englewood Cliffs, Prentice-Hall (1975)

79. Nadirashvili, N.: Rayleigh's conjecture on the principal frequency of the clamped plate. Arch. Ration. Mech. Anal. 129, 1-10 (1995) 
80. Osserman, R.: Isoperimetric inequalities and eigenvalues of the Laplacian. In: Proceedings of the International Congress of Mathematicians (Helsinki, 1978), pp. 435-442, Acad. Sci. Fennica, Helsinki (1980)

81. Payne, L.E.: A note on inequalities for plate eigenvalues. J. Math. Phys. 39, 155-159 (1960/1961)

82. Payne, L.E.: Isoperimetric inequalities and their applications. SIAM Rev. 9, 453-488 (1967)

83. Payne, L.E., Pólya, G., Weinberger, H.F.: Sur le quotient de deux fréquences propres consécutives. Comptes Rendus Acad. Sci. Paris 241, 917-919 (1955)

84. Payne, L.E., Pólya, G., Weinberger, H.F.: On the ratio of consecutive eigenvalues. J. Math. Phys. 35, 289-298 (1956)

85. Payne, L.E., Weinberger, H.F.: An optimal Poincaré inequality for convex domains. Arch. Ration. Mech. Anal. 5, 286-292 (1960)

86. Pleijel, Å.: A study of certain Green's functions with applications in the theory of vibrating membranes. Ark. Math. 2, 553-569 (1954)

87. Pólya, G., Szegö, G.: Isoperimetric Inequalities in Mathematical Physics. Princeton University Press, Princeton, NJ (1951)

88. Rayleigh, J.W.S.: The Theory of Sound, 2nd edn. revised and enlarged (in 2 vols.), Dover Publications, New York (1945) (republication of the 1894/1896 edition)

89. Reed, M., Simon, B.: Methods of Modern Mathematical Physics, vol. 3. Academic Press, NY (1979)

90. Reed, M., Simon, B.: Methods of Modern Mathematical Physics Vol. 4. Analysis of Operators. Academic Press, NY (1978)

91. Riemann, B.: Über die Anzahl der Primzahlen unter einer gegebenen Grösse, Monatsberichte der Berliner Akademie, pp. 671-680 (1859)

92. Singer, I.M., Wong, B., Yau, S.-T., Yau, S.S.-T.: An estimate of the gap of the first two eigenvalues in the Schrödinger operator. Ann. Scuola Norm. Sup. Pisa Cl. Sci. 12(4 ), 319-333 (1985)

93. Sridhar, S., Kudrolli, A.: Experiments on not "Hearing the Shape" of drums. Phys. Rev. Lett. 72, 21752178 (1994)

94. Sunada, T.: Riemannian coverings and isospectral manifolds. Ann. Math. 121, 169-186 (1985)

95. Szegö, G.: On membranes and plates. Proc. Nat. Acad. Sci. USA 36, 210-216 (1950)

96. Szegö, G. : On the vibrations of a clamped plate. In: Atti del Quarto Congresso dell'Unione Matematica Italiana, Taormina, 1951, vol. II, pp. 573-577. Casa Editrice Perrella, Roma (1953)

97. Szegö, G.: Inequalities for certain eigenvalues of a membrane of given area. J. Ration. Mech. Anal. 3, 343-356 (1954)

98. Szegö, G.: Note to my paper "On membranes and plates". Proc. Nat. Acad. Sci. USA 44, 314316 (1958)

99. Talenti, G.: On the first eigenvalue of the clamped plate. Ann. Mat. Pura Appl. 129(4), 265-280 (1981)

100. Talenti, G.: Elliptic equations and rearrangements. Ann. Scuola Norm. Sup. Pisa 3(4), 697-718 (1976)

101. Thomas, I.: Greek Mathematics. In: Newman J.R. (ed.) The World of Mathematics, vol. 1, pp. 189-209, Dover, NY (2000)

102. Vergil: The Aeneid, [English translation by Sarah Ruden], Yale University Press, New Haven, CT (2008)

103. Weinberger, H.F.: An isoperimetric inequality for the n-dimensional free membrane problem. J. Ration. Mech. Anal. 5, 633-636 (1956)

104. Weyl, H.: Über die asymptotische Verteilung der Eigenwerte. Nachr. Akad. Wiss. Göttingen Math. Phys., Kl. II, 110-117 (1911)

105. Weyl, H.: Ramifications, old and new, of the eigenvalue problem. Bull. Am. Math. Soc. 56, 115139 (1950)

106. Yau, S.-T.: Nonlinear analysis in geometry, Monographies de LEnseignement Mathématique, vol. 33. Série des Conférences de lUnion Mathématique Internationale, vol. 8, Geneva (1986)

107. Yu, Q.-H., Zhong, J.-Q.: Lower bounds of the gap between the first and second eigenvalues of the Schrödinger operator. Trans. Am. Math. Soc. 294, 341-349 (1986)

108. Zelditch, S.: Spectral determination of analytic bi-axisymmetric plane domains. Geom. Funct. Anal. 10, 628-677 (2000)

109. Zelditch, S.: Inverse spectral problems for analytic domains II: $\mathbb{Z}^{2}$-symmetric domains. Ann. Math. 170, 205-269 (2009) 